\title{
2 Nonlinear dynamics of the CAM circadian rhythm in response to 3 environmental forcing
}

4 by Samantha Hartzell ${ }^{1}$, Mark S. Bartlett ${ }^{2}$, Lawrence Virgin ${ }^{3}$, Amilcare Porporato $^{4}$

5

6

7

8

9

10

11

12
Corresponding Author:

Amilcare Porporato

Department of Civil and Environmental Engineering

Duke University

Durham, NC 27708 U.S.A.

Phone +1919660 5511

Fax +1919660 5219

e-mail amilcare@duke.edu

${ }^{1}$ Civil and Environmental Engineering, Duke University, Durham, NC 27708; e-mail samhartz@mit.edu

${ }^{2}$ Civil and Environmental Engineering, Duke University, Durham, NC 27708; e-mail Mark.Bartlett@duke.edu

${ }^{3}$ Mechanical Engineering and Materials Science, Duke University, Durham, NC 27708; e-mail l.virgin@duke.edu

${ }^{4}$ Civil and Environmental Engineering, Duke University, Durham, NC 27708; e-mail amilcare.porporato@duke.edu 
14 Crassulacean acid metabolism (CAM) photosynthesis functions as an endogenous cir15 cadian rhythm coupled to external environmental forcings of energy and water availability.

16 This paper explores the nonlinear dynamics of a new CAM photosynthesis model [2] and 17 investigates the responses of CAM plant carbon assimilation to different combinations of en18 vironmental conditions. The CAM model [2] consists of a Calvin cycle typical of C3 plants 19 coupled to an oscillator of the type employed in the Van der Pol and FitzHugh-Nagumo 20 systems. This coupled system is a function of environmental variables including leaf tem21 perature, leaf moisture potential, and irradiance. Here, we explore the qualitative response 22 of the system and the expected carbon assimilation under constant and periodically forced 23 environmental conditions. The model results show how the diurnal evolution of these vari24 ables entrains the CAM cycle with prevailing environmental conditions. While constant 25 environmental conditions generate either steady-state or periodically oscillating responses in 26 malic acid uptake and release, forcing the CAM system with periodic daily fluctuations in 27 light exposure and leaf temperature results in quasi-periodicity and possible chaos for certain 28 ranges of these variables. This analysis is a first step in quantifying changes in CAM plant 29 productivity with variables such as the mean temperature, daily temperature range, irradi30 ance, and leaf moisture potential. Results may also be used to inform model parametrization 31 based on the observed fluctuating regime.

33 KEY WORDS: crassulacean acid metabolism (CAM) photosynthesis, circadian rhythm os34 cillator, chaos, quasi-periodicity, bifurcation

\section{1. Introduction}

36 Because of their high water use efficiency and often succulent nature, crassulacean acid 37 metabolism (CAM) plants thrive in environments where water is limiting, achieving a greater 38 net $\mathrm{CO}_{2}$ uptake per leaf area than their $\mathrm{C} 3$ and $\mathrm{C} 4$ counterparts [39]. Their greater water use 39 efficiency makes CAM plants a potentially important contributor to sustainable agriculture 40 in arid and semiarid regions of the world. While the modelling of CAM is still in its infancy 
$41[45,34,4,5,6,47]$, a comprehensive and faithful model of CAM photosynthesis coupled to 42 local climate conditions would allow for more accurate estimates of CAM productivity and 43 the associated crop yield in arid and semiarid regions. A recent model [2] attempts to couple 44 CAM photosynthesis to climatic conditions. Here we explore this model to better understand 45 the resulting carbon assimilation predictions under conditions of external environmental 46 forcing.

47 The modeling of CAM is challenging because the exact functioning of CAM is not com48 pletely known. Nevertheless the model of [2] moves forward based on the assumption that 49 the CAM circadian rhythm functions through a combination of mechanisms, including the 50 regulation of enzymes such as $\mathrm{RuBisCO}$ and $\mathrm{PEPC}$, gene expression, the cytoplasmic $\mathrm{pH}$ 51 and vacuolar malic acid levels, and the state of the cell vacuole membrane [32]. The overall 52 effect of these mechanisms is assumed to produce a relaxation oscillation consisting of a slow 53 accumulation or release of malic acid followed by a more rapid change in the overall state 54 of the plant (enzyme levels, gene expression, the cytoplasmic pH and vacuolar malic acid 55 levels, etc.). Such oscillations are ubiquitous in biology, especially in descriptions of circa56 dian rhythms. Indeed, archetypical relaxation oscillators such as the Van der Pol system 57 have been used to analyze the circadian rhythm of the human core temperature as well as 58 contractions of the human heart [7, 49]. The circadian rhythm oscillator model formulated 59 in [2] consists of two nonlinear differential equations, the first of which describes the overall 60 circadian state of the plant and the second of which includes a photosynthesis model for the 61 influx and efflux of carbon $\left(\mathrm{CO}_{2}\right)$ from the cell vacuole of the CAM plant. This new system, 62 while affected by changes in external conditions due to dependencies on light, leaf temper63 ature, and leaf potential, also reproduces the endogenous rhythm of CAM that persists in 64 continuous light conditions $[36,56]$. This work aims to understand the new system under 65 constant and periodic environmental forcings and to better understand the rates of carbon 66 assimilation predicted by the model.

67 After a brief overview of CAM, Section 2 presents an outline of the coupled system of 68 equations, one describing the circadian state of the system and the other describing the malic 69 acid concentration in the vacuole, which together determine the circadian rhythm. We then 
74 Appendices.

\section{2. CAM model}

76

97 I under well-watered conditions [57]. 
99 The circadian rhythm of $\mathrm{CO}_{2}$ uptake and release is modelled following [2], and consists of a 100 coupled system of nonlinear differential equations for $z$, which summarizes the global effect 101 of plant enzyme activity, gene expression, and vacuolar activity, and $M$, which represents 102 the concentration of malic acid in the cell vacuole. The first nonlinear differential equation of 103 the CAM circadian rhythm oscillator is for the overall circadian state of the plant described 104 by the order variable $z$ and given by

$$
t_{r} \frac{d z}{d t}=\frac{M-M_{E}\left(z, T_{l}\right)}{M_{\max }}
$$

105 where $t_{r}$ is the relaxation time, which controls the rate of change in $z$ (see Section 3 ). At low $106 z$ values (less than 0.5), malic acid is being stored, while at high $z$ values (greater than 0.5 ) 107 malic acid is being released and utilized. The nonlinear function $M_{E}\left(z, T_{l}\right)$ represents an 108 equilibrium concentration of malic acid as a function of the circadian state, $z$, and the leaf 109 temperature, $T_{l}$ (see Appendix F). The value of $M_{\max }$ is equivalent to the maximum equilib110 rium concentration of malic acid at the low temperature $T_{L}$, i.e., $M_{\max }=\max \left[M_{E}\left(z, T_{L}\right)\right]$, 111 and functions to normalize $z$.

112 The second nonlinear differential equation describes the rate of change in the concentra113 tion of malic acid in the cell vacuole, $M$, as

$$
L_{M} \frac{d M}{d t}=A_{s v}\left(M, z, T_{l}, \psi_{l}, \phi\right)+R_{d v}\left(T_{l}, \phi\right)-A_{v c}\left(M, z, T_{l}, \phi\right)
$$

114 where $L_{M}$ is the ratio of malic acid storage volume to the carbon flux surface area and has 115 units of length. The terms $A_{s v}, R_{d v}$, and $A_{v c}$ represent the carbon fluxes to and from the 116 cell vacuole: $A_{s v}$ represents the carbon flux from the stomatal cavity, which is responsible 117 for exchange of gases with the atmosphere; $R_{d v}$ represents the flux from the dark respiration 118 node, where breakdown of carbohydrates releases $\mathrm{CO}_{2}$ to either the Calvin cycle $\left(R_{d c}\right)$ or the 119 cell vacuole $\left(R_{d v}\right)$; and $A_{v c}$ represents the flux of carbon to the site of carbon assimilation, 120 i.e., the Calvin cycle (see Fig. 1 and Appendix A). These functions depend nonlinearly on 
121 the system variables $M$ and $z$, as well as the external forcing from the leaf temperature, $T_{l}$, 122 the leaf water potential, $\psi_{l}$, and the light intensity, $\phi$.

123 The carbon flux from the stomata to the cell vacuole is modeled as [2]

$$
A_{s v}=\left(A_{s v, \max }\left(T_{l}\right)-R_{d v}\left(T_{l}, \phi\right)\right) \times f_{\psi_{l}}\left(\psi_{l}\right) \times f_{O}(z) \frac{\left(M_{S}\left(T_{l}\right)-M\right)}{\left(\alpha_{2} M_{S}\left(T_{l}\right)+M_{S}\left(T_{l}\right)-M\right)},
$$

124 where $A_{s v, \max }$ represents the overall maximum rate of carbon flux to the cell vacuole (see 125 Appendix E), $R_{d v}$ represents the carbon supplied by dark respiration (see Appendix C),

$126 f_{O}(z)$ represents the circadian control of the flux (see Appendix G), and $\frac{\left(M_{S}\left(T_{l}\right)-M\right)}{\alpha_{2} M_{S}\left(T_{l}\right)+M_{S}\left(T_{l}\right)-M}$ 127 is a Michaelis-Menten type term representing the malic acid storage limitation based on the 128 concentration of malic acid that the system can store, $M_{S}$ (see Appendix $\mathrm{H}$ ), where $\alpha_{2}$ is a 129 parameter between 0 and 1 . The function $f_{\psi_{l}}$ accounts for the effects of water stress, and is 130 dependent on $\psi_{l}$, the leaf water potential (see Appendix D).

131 The carbon flux from the cell vacuole to the carbon cycle is given by

$$
A_{v c}=\left(A_{\phi, c_{c}, T_{l}}-R_{d c}\left(T_{l}, \phi\right)\right) \times\left(1-f_{O}(z)\right) \frac{M}{\alpha_{1} M_{\max }+M},
$$

132 where $A_{\phi, c_{c}, T_{l}}$ represents the $\mathrm{CO}_{2}$ demand (see Appendix $\mathrm{B}$ ) and $R_{d c}$ is the flux of carbon 133 from dark respiration to the Calvin cycle (see Appendix C). These functions describe pho134 tosynthesis and are dependent on light intensity, mesophyll cytosol $\mathrm{CO}_{2}$ concentration $c_{c}$, 135 and leaf temperature. The mathematical formulation for the Calvin cycle demand, given 136 in Appendices A-C, follows well known photosynthesis models [15, 13]. The order function $1371-f_{O}(z)$ is a sigmoidal curve ranging from 0 to 1 which describes the relative rate of malic 138 acid diffusion from the cell vacuole to the cytoplasm and the overall activation state of the 139 decarboxylation enzymes, and $\frac{M}{\alpha_{1} M_{\max }+M}$ is the Michaelis-Menten term representing how the 140 supply of malic acid in the vacuole limits the malic acid diffusion and decarboxylation, where $141 \alpha_{1}$ is a parameter between 0 and 1.

142 The CAM system can be written as a single second order differential equation for the 
143 circadian order, $z$, in the form of a generalized classical Liénard equation,

$$
t_{r} M_{\max } \frac{d^{2} z}{d t^{2}}+M_{E}^{\prime}\left(z, T_{l}\right) \frac{d z}{d t}+g\left(\frac{d z}{d t}, z, T_{l}, \psi_{l}, \phi\right)=0
$$

144 where $M_{E}^{\prime}\left(z, T_{l}\right)=\frac{d}{d t} M_{E}\left(z, T_{l}\right)[10,2$, p. 130], and

$$
g\left(\frac{d z}{d t}, z, T_{l}, \psi_{l}, \phi\right)=-\frac{1}{L_{M}}\left(A_{s v}\left(\frac{d z}{d t}, z, T_{l}, \psi_{l}, \phi\right)+R_{d v}\left(T_{l}, \phi\right)-A_{v c}\left(\frac{d z}{d t}, z, T_{l}, \phi\right)\right)
$$

145 The major nonlinearities in the system arise from the circadian order function, $f_{O}(z)$, in Eqs.

1463 and 4, from the Michaelis Menten dependence on $M$ in Eqs. 3 and 4, and from the malic 147 acid equilibrium equation $M_{E}\left(z, T_{l}\right)$ in Eq. 1, which is a cubic curve typical of relaxation

148 oscillations such as the FitzHugo-Nagumo or Van der Pol models. Additive periodic forcing 149 of the Liénard equation has been described in the literature [50, 10] but this differs from 150 the more complex forcing induced, in our case, by the environmental variables $T_{l}, \phi$, and $\psi_{l}$, 151 which act through the multiplicative functions of $M_{E}^{\prime}$ and $g$.

152 2.2 Model Parametrization

153 The model parameters are for Agave tequilana, a CAM plant native to Jalisco, Mexico, which 154 is commonly used in tequila production (see Table 2). Although many of the parameters 155 are specific to the plant Agave tequilana, the main features of the circadian rhythm of other 156 CAM plant species is expected to be of a similar form and have a similar response to external 157 parameters.

\section{3. Model behavior under constant environmental conditions}

159 The model is studied first under constant environmental conditions (constant temperature, 160 light, and leaf water potential) as a basis for better understanding its response to periodically 161 forced environmental conditions intended to roughly model the day-night cycle (see Section

162 4). Under conditions of constant light and temperature considered in this section, the system 163 may undergo supercritical Hopf bifurcation from a stable fixed point to a limit cycle. 
165 The system oscillates in the $(z, M)$ phase plane around the fixed point that lies at the 166 intersection of the system nullclines for $d M / d t$ and $d z / d t$ (see Figs. 2 and 3). The nullcline $167 d z / d t=0$ is given by

$$
\frac{M}{M_{\max }}=\frac{M_{E}\left(z, T_{l}\right)}{M_{\max }}
$$

168 and identifies where changes in the system are due only to changes in $M$ (Figs. 2 and 3). 169 The shape of this nullcline changes depending on the leaf temperature and various nullclines 170 are displayed between the low leaf temperature of $T_{L}$ and high leaf temperature of $T_{H}$ (see 171 Fig. 2). The nullcline $d M / d t=0$ identifies where changes in the system are due only to 172 changes in $z$ and is given by

$$
A_{s v}\left(M, z, T_{l}, \psi_{l}, \phi\right)+R_{d v}\left(T_{l}, \phi\right)-A_{v c}\left(M, z, T_{l}, \phi\right)=0 .
$$

173 The shape of this nullcline varies depending on the leaf potential, $\psi_{l}$, and two nullclines are 174 displayed for $\psi_{l 1}$ and $\psi_{l 0}$ (Fig. 2). An example of an oscillation around the fixed point for $175 T_{l}=293.15 \mathrm{~K}$ and $\psi_{l}=-0.5 \mathrm{MPa}$ is shown in Figure 3e.

176 As is typical of a relaxation oscillation, the system is characterized by two widely sepa177 rated time scales. The longer time scale characterizes the slower filling and emptying of the 178 cell vacuole with malic acid, while the shorter time scale characterizes the more rapid change 179 in circadian order and is controlled by the relaxation time, $t_{r}$ (Eq. 1). For a relatively small 180 value of $t_{r}$, i.e. 1 minute (Fig. 3a), the fast response in $d z / d t$ causes the malic acid concen181 tration to closely follow the thermodynamic equilibrium value defined by the nullcline. An 182 increase in the relaxation time slows the transition of $z$ and allows a further accumulation 183 and release of malic acid. As the relaxation time continues to increase to larger values, the 184 unstable fixed point of the limit cycle transitions to a stable fixed point for the system (Fig. $1853 b)$. 
187 The effect of leaf temperature on the circadian rhythm for non-water stressed conditions

188 (a leaf potential of $-0.5 \mathrm{MPa}$ ) is shown in Figure 3c, while the effect of leaf potential (at 189 constant temperature $293.15 \mathrm{~K}$ ) is shown in Figure $3 \mathrm{~d}$. As the leaf temperature, $T_{l}$, increases 190 past the low temperature, $T_{L}$ (see Table 1 ), the circadian system undergoes a supercritical 191 Hopf bifurcation [53]. As the leaf temperature increases further, the oscillations of this limit 192 cycle become smaller until, at a critical temperature, the system trajectory spirals towards a 193 stable fixed point characterized by a moderate to high circadian order, $z$, and low malic acid 194 concentration (an empty cell vacuole). This transition occurs when the slope of the nullcline 195 (for $d z / d t=0$ ) changes from a positive value to a negative value. A limit cycle with the 196 corresponding nullclines, fixed point, and vector field is displayed in Figure 3e.

197 The hydrologic cycle also influences these circadian oscillations through the leaf potential 198 parameter (Fig. 3d). Leaf moisture potential, $\psi_{l}$, is dependent on soil moisture and high 199 negative values (e.g., in this paper near -3.0 MPa) are typically considered to be an indicator 200 of plant water stress under drought conditions. As the level of water stress increases, i.e., 201 the leaf potential decreases, the photosynthesis model becomes impaired, so the oscillations 202 of the system become smaller. As a result, the maximum value of malic acid concentration 203 decreases, narrowing the limit cycle (Fig. 3d). This decrease happens rapidly around the 204 leaf potential value of $\psi_{l}=-3 \mathrm{MPa}$, which is the threshold below which the maximum plant 205 water stress occurs in the model (see $\psi_{l_{1}}$ in Table 1$)$.

2063.3 Bifurcations, period length, and carbon assimilation

207 Having noted how temperature and leaf potential impact the circadian rhythm cycle, we now 208 consider the overall behavior of the circadian oscillation and carbon assimilation in response 209 to a range of temperatures and leaf potentials (Fig. 4). At very low leaf temperatures (below $210283.15 \mathrm{~K}$ ), there is a fixed point which corresponds to a state with a low value of circadian 211 order and a full vacuole (Fig. 4b). At $283.15 \mathrm{~K}$, the system undergoes a supercritical Hopf 212 bifurcation to a stable limit cycle. As the temperature increases from $283.15 \mathrm{~K}$, the period 
213 length decreases and the oscillations of $z$ become increasingly closer together (Fig. 4b).

214 Finally, after the leaf temperature increases past $300.5 \mathrm{~K}$, there is a second Hopf bifurcation 215 and the system goes to a stable fixed point (Fig. 4b) where $M$ is at a low value and $z$ is 216 at a moderate value. Between 300.5-302.15 $\mathrm{K}$ (the value of $T_{H}$ ), $\mathrm{CO}_{2}$ is both entering and 217 leaving the vacuole, with a very small amount of malic acid being maintained in the vacuole. 218 This temperature $(300.5 \mathrm{~K})$ marks the beginning of the breakdown of the CAM cycle under 219 constant light and temperature conditions (as we will describe later, this type of fixed point 220 is unique to constant conditions and disappears under very small forcings of either light or 221 temperature). Finally, at $T_{H}=302.15 \mathrm{~K}$, the stable fixed point transitions from a moderate 222 to a high value of circadian order, $z$. Here, the vacuole empties completely and carbon flux to 223 the vacuole ceases (Fig. 4c). The location of the bifurcation points agree well with previous 224 results that show the maintenance of the CAM circadian rhythm over the range $T_{L}=283.15$ $225 \mathrm{~K}$ to $T_{H}=302.15 \mathrm{~K}[18,9,12,41]$.

226 The model parameters for this study are consistent for average nocturnal and diurnal 227 temperatures of $293.15 \mathrm{~K}$ and $303.15 \mathrm{~K}$, respectively, and within this temperature range a 228 model period of around 24 hrs. is found (Fig. 4a). The period length around 24 hours is 229 consistent with studies of other circadian rhythms which find that under conditions with no 230 external cues, the free-running period is generally close to, but not necessarily equal to, 24 231 hours $[8,48]$. In this model, as the temperature increases and the oscillations of $z$ become 232 smaller, the period length of the CAM cycle decreases and the oscillations become more 233 rapid (Fig. 4a). At a low temperature of $283 \mathrm{~K}$, the period length is around 60 hours, while 234 at a high temperature of $302 \mathrm{~K}$ the period is less than 10 hours. It is remarkable that within 235 this temperature range (283-302 K) a period length of 24 hrs. is obtained naturally (i.e., 236 without calibration) by the complex model proposed in [2] using parameter values obtained 237 from published data.

238 Though a period of around $24 \mathrm{hrs}$. was found for the average nocturnal and diurnal 239 temperatures of $293.15 \mathrm{~K}$ and $303.15 \mathrm{~K}$, respectively, this period will vary as the average 240 temperature range changes. This occurs in part because CAM plants acclimate to the 241 prevailing average environmental conditions, and they have significant differences in the 
242 rates of nocturnal carbon uptake as represented by $A_{s v, \max }\left(T_{l}\right)$ and diurnal carbon uptake

243 for direct carbon assimilation as represented by $A_{\phi, c_{i}, T_{l}}$ for different average temperatures $[2$,

244 p. 122]. We account for such acclimation with an exponential increase/decline in $A_{s v, \max 0}$ 245 and $A_{\phi, c_{c}, T_{l}}$ given by the factor

$$
f_{a}\left(T_{l, a v g}\right)=b_{1} \exp \left(-b_{2}\left(T_{l, a v g}-T_{L}\right)\right)
$$

246 where $T_{l, a v g}$ is the average daily temperature over multiple days, $T_{L}$ represents the low end 247 of the temperature range of CAM (Table 1 ), and $b_{1}$ and $b_{2}$ are parameters specific to the 248 experimental data of a certain plant species parameters chosen to fit the data (Table 1). 249 For the constant temperaure conditions explored here, $T_{l, a v g}$ is simply the constant leaf 250 temperature $T_{l}$. Available experimental data for the period of CAM is for plants that are 251 acclimated to certain average nocturnal and diurnal temperatures. To compare the period of 252 the model to this data, we use a slightly increased temperature range of CAM, so the values 253 of $T_{L}$ and $T_{H}$ for this comparison are $278 \mathrm{~K}$ and $304 \mathrm{~K}$, respectively. This experimental data 254 describes an increase in period length with an increase in temperature [56, 18], and based on 255 the acclimation function, $f_{a}\left(T_{l, a v g}\right)$, the period of the model agrees well with the data (Fig. $2565)$.

257 The advantage of CAM is the water efficient productivity from the carbon flux $A_{s v}$, which 258 is a measure of the amount of carbon taken up and fixed as malic acid at night when water 259 losses are typically lower (Fig. 4c, f). Note that this flux is only a portion of the total 260 carbon assimilation and does not include the less water efficient direct carbon assimilation 261 fluxes of C3 photosynthesis that primarily occur during Phases II and IV. As expected, the 262 flux $A_{s v}$ is 0 at the stable fixed point, which occurs at low temperatures below $283.15 \mathrm{~K}$, 263 but when $T_{l}$ increases past $283.15 \mathrm{~K}$, the assimilation flux rapidly increases to values of 264 about $200 \mathrm{mmol} / \mathrm{m}^{2} /$ day, reaching a maximum value of $220 \mathrm{mmol} / \mathrm{m}^{2} /$ day at $293 \mathrm{~K}$. As the 265 temperature increases and moves out of this optimal range, the assimilation decreases. A 266 sharp spike appears at $301 \mathrm{~K}$, which corresponds to the breakdown of the CAM cycle when 267 the system transitions to a stable fixed point between the 300.5-302.5 K (Fig. 4b). This fixed 
point, which occurs only under conditions of no forcing or very little forcing, arises when

269 malic acid is simultaneously being taken up and fixed, and is a transitional state before the 270 model terminates the CAM cycle.

271 Variations in the leaf potential between the allowed values of -3.0 and $-0.5 \mathrm{MPa}$ have 272 an equally important effect on the period length and associated carbon assimilation, but a 273 smaller effect on the amplitude of the oscillations (Fig. 4d-f). As the leaf potential becomes

274 less negative, i.e., water stress decreases, the values of the circadian order, $z$, shift upward 275 slightly, but a similar limit cycle is maintained for all leaf potential values greater than -3.0 $276 \mathrm{MPa}$ (Fig. 4e). The free-running period is, however, greatly affected by changes in the leaf 277 potential, and may vary from 20 to upwards of 80 hours in an almost exponential manner 278 (Fig. 4d). Although no experimental data are available on the effect of leaf moisture potential 279 on CAM period length, the carbon assimilation responds to changes in leaf moisture in an 280 expected manner; as $\psi_{l}$ approaches the maximum allowed water stress, the flux $A_{s v}$ decreases 281 and the carbon assimilation through the CAM pathway approaches 0 , thereby increasing the 282 period length (Fig. 4f). It is noteworthy that, again, an endogenous circadian oscillation 283 centered around 24 hours is obtained using estimated parameters without any calibration; 284 this result lends support that the various complex parameters are faithfully modelled and 285 coupled in a realistic way.

\section{4. Effects of environmental periodic forcing}

287 The behavior of the system is further examined with periodic forcings of temperature and 288 light to simulate the behavior of CAM plants under the natural daytime and nighttime 289 fluctuations observed in the environment. Here, we choose a simplistic description for the 290 temperature, modelling it as a sine wave which increases to a maximum value at noon and 291 decreases to a minimum value at midnight, i.e.,

$$
T_{l}(t)=T_{l, a v}+A \sin (\omega t-\pi / 2),
$$


292 where $A(\mathrm{~K})$ represents the displacement of the temperature from the mean $\left(T_{l, a v}\right), \omega$ is $2932 \pi / p, p=24$ hours, and $t$ is time (hrs). While there are various methods of interpolating 294 temperature data from maximum and minimum daily values, any one of which could easily 295 be coupled to this model [51], this equation is one of the simplest approximations, which has 296 previously been used with respect to plant productivity [58]. The solar radiation is explored 297 using a piecewise function alternating between 0 at night and a parabola during the day [27], 298 i.e.,

$$
\phi(t)= \begin{cases}0 & \text { for } t \bmod 24<12 \\ \frac{\phi_{h}}{36}\left(-(t \bmod 24)^{2}+24(t \bmod 24)-648\right) & \text { for } t \bmod 24 \geq 12\end{cases}
$$

299 where $\phi(\mathrm{t})$ is the solar radiation $\left(\mathrm{W} / \mathrm{m}^{2}\right), \phi_{h}$ is the maximum solar radiation, $\omega$ is $2 \pi / p$, $300 p=24$ hours, and $t$ is time (hrs). Responses typical to laboratory conditions, i.e. 12 301 hours of light and 12 hours of darkness, were also explored. The results were found to 302 vary qualitatively from the more realistic light forcing results shown here, suggesting that 303 laboratory experiments which analyze CAM circadian rhythms using such conditions may 304 not be the best predictors for behavior under natural conditions.

\subsection{Bifurcations}

306 Forcing the leaf temperature to simulate typical daily conditions has a strong effect on the 307 CAM cycle (Fig. 6). When there is a small degree of temperature forcing, the periodic 308 limit cycle at $300 \mathrm{~K}$ becomes unstable and quasi-periodic or chaotic behavior is observed for 309 forcing amplitudes less than $2.7 \mathrm{~K}$. At $2.7 \mathrm{~K}$, the system returns to periodicity, but this time 310 with a much larger amplitude (Fig. 6a). Behavior for forcing amplitudes between 1 and $3112.5 \mathrm{~K}$ are shown in the inset in Figure 7a, and the transition to chaos or quasiperiodicity 312 observed for $A \leq 1 K$ seems to arise through the period doubling mechanism. When leaf 313 temperature is forced with an amplitude of $1 \mathrm{~K}$, which generates a $2 \mathrm{~K}$ range between the 314 maximum and minimum temperatures (Fig. 6c), the response shows many similarities to the 315 unforced version in Figure 4b, but the stable fixed points have disappeared and are replaced 316 by large oscillations (below $T_{L}$ ) and small oscillations (above $T_{H}$ ). Also of note are several 317 windows which appear either chaotic or quasi-periodic. Samples from two of these windows 
318 are displayed in Figure $7 \mathrm{~b}$ and $\mathrm{c}$ for more detail. For a leaf temperature amplitude of 5

$319 \mathrm{~K}$ (Fig. 6e), an average temperature between 286 and $288.5 \mathrm{~K}$ results in quasi-periodic or 320 chaotic behavior where there seems to be some period doubling, but the system then returns 321 to a periodic oscillation for higher temperature values, except for a brief transition at 301 322 to $302 \mathrm{~K}$. On the other hand, a leaf temperature amplitude of $10 \mathrm{~K}$ (Fig. 6g), as would 323 be seen in a typical desert environment, results in a periodic solution for the entire range 324 of average temperatures between 283 and $302 \mathrm{~K}$. As the average temperature increases past $325285 \mathrm{~K}$, there is a jump in the solution and $z$ shifts to lower values, which occurs due to 326 the changing rates of malic acid uptake $\left(A_{s v, \max }\left(T_{l}\right)\right)$ and release $\left(A_{\phi, c_{i}, T_{l}}\right)$ that both have 327 a leaf temperature dependency. Because, under this forcing regime, the temperatures dip 328 significantly below $T_{L}, z$ becomes slightly negative in some instances; however, this behavior 329 is inconsequential as the state of the system is determined by whether $z$ is greater than or 330 less than 0.5 , as set by the order function, $f_{O}(z)$. Overall, when the temperature is varied 331 significantly, the amplitude of the resulting oscillations of $z$ becomes nearly the same over 332 a similar range of average temperatures, unlike the behavior seen when the temperature is 333 held constant or varied only slightly. This similarity in the oscillation of $z$ leads to a more 334 consistent carbon assimilation over a wider range of average temperatures.

335 This response to changes in temperature has an important effect on $\mathrm{CO}_{2}$ assimilation 336 via the CAM pathway, and interesting behavior begins to emerge when the amplitude of 337 leaf temperature fluctuations allows the temperature to cross the bifurcation value of 300.5 $338 \mathrm{~K}$ during the course of the day (Fig. 6b). For small $A$ less than $2 \mathrm{~K}$, the leaf temperature 339 remains near $300 \mathrm{~K}$ in a range of temperature where carbon assimilation is optimal (Fig. 340 4c). As soon as $A$ becomes larger than $2 \mathrm{~K}$, however, the temperature jumps into the range 341 where the CAM cycle shuts down (Fig. 4c). Consequently, there is a dramatic decrease in 342 the average carbon assimilation (Fig. 6b). However, as the amplitude increases further past $3432 \mathrm{~K}$, the carbon assimilation improves, reaching moderate values by $A=15 K$. For $A>2 K$, 344 the last traces of the stable fixed point disappear, and carbon assimilation via the CAM 345 pathway is maintained for the entire range of average temperatures up to $T_{l}=305 K$. This 346 behavior suggests that, under conditions of high mean temperatures, a larger temperature 
347 range, such as that observed in most arid environments, is beneficial for CAM functioning.

348 Such a conclusion is supported by experimental studies which have found that the optimal

349 nighttime temperature is 10 to $20 \mathrm{C}$, while the optimal daytime temperature is above $20 \mathrm{C}$ $350[17]$.

351 Periodic forcing of the irradiance also has a significant effect on the system's functioning,

352 which is shown here for different temperature inputs, i.e., constant and forced (Fig. 8).

353 Varying the maximum light input, $\phi_{h}$, has essentially no effect when $\phi_{h}$ is above the thresh-

354 old value of $100 \mathrm{~W} / \mathrm{m}^{2}$, where the leaf temperature and leaf potential are the controlling

355 factors. Since typical maximum light values, $\phi_{h}$, are much greater than this threshold, the

356 response of the plant under a periodically forced light input depends much more on the leaf

357 temperature and water potential than it does on the irradiance. For a maximum light input

358 at a typical environmental value of $500 \mathrm{~W} / \mathrm{m}^{2}$ and a nighttime light input of $0 \mathrm{~W} / \mathrm{m}^{2}$, the

359 system exhibits possible chaotic behavior for constant temperatures below about $291.2 \mathrm{~K}$.

360 It then maintains a periodic solution for constant temperatures above this threshold (Fig.

361 8a). On the other hand, forcing both the light and temperature simultaneously (with the

362 temperature amplitude equal to $5 \mathrm{~K}$ ), as in Figure 8c, results in mainly periodic solutions.

363 As in the case with temperature forcing, both stable fixed points become unstable when the

364 light is varied diurnally.

365 Unlike temperature forcing, irradiance forcing alone does not extend the range of mean 366 temperatures under which CAM photosynthesis is expected to function (Fig. 8b). In fact, 367 when compared with the unforced system, the forced system is expected to stop functioning 368 at a slightly lower temperature (301.5 K compared with $302.15 \mathrm{~K}$ ). This behaviour occurs 369 because the periodic cessation of light does not allow for the fixed point that existed under 370 constant irradiance and between the leaf temperatures of 300.5 to $302.15 \mathrm{~K}$, which was not 371 a realistic state. When the solar radiation is forced diurnally, a clear optimal temperature 372 emerges around $291 \mathrm{~K}$ (Figs. 8b, d). This optimal temperature agrees with observed ranges 373 of optimal temperatures for CAM photosynthesis (P. Inglese, personal communication). Al374 though the maximum assimilation is lessened when temperature forcing is added to the 375 picture (Fig. 8d), the optimal average temperature remains the same. The fact that the op- 
timal temperatures in the model agree with observed ranges suggests that the model may be

377 used to predict optimal combinations of environmental conditions in different environments.

378 As expected, forcing of the temperature, light, or both, always results in entrainment

379 of the CAM cycle to a 24 hour cycle in regions where the response is periodic. This phase

380 locking is an important characteristic of circadian rhythms. Such rhythms are known to

381 deviate from a 24-hour period in the absence of a light-dark cycle, but are entrained by

382 external forcing of environmental conditions, as long as this forcing occurs with a period

383 close to 24 hours [48].

3844.2 Poincaré section and spectral analysis

385 Poincaré sections and spectral analysis can both be used to distinguish between periodic 386 and chaotic systems. The Poincaré section for a periodically driven system can be obtained 387 by sampling the $3 \mathrm{D}$ phase portrait at periodic intervals and shows the instances where the 388 trajectory crosses a plane in the phase space from one direction [1]. Another method of 389 distinguishing between periodicity (or quasi-periodicity) and chaos involves computing the 390 fast Fourier transform (FFT) of the time series. The FFT of a periodic system will show 391 a small number of frequencies above a certain cutoff frequency, while the FFT of a chaotic 392 system will show a much larger number of frequencies above the cutoff value [55].

393 Figures 9a and 9b show a time series and 3D phase portrait, respectively, in conditions 394 of periodic temperature forcing with an average temperature of $298 \mathrm{~K}$ and an amplitude of 1 $395 \mathrm{~K}$. The time series shows the evolution of the system circadian order with time while the 3-D 396 phase portrait shows the evolution in time of $z, M$, and $T_{l}$. Unlike the regular oscillations 397 which occur under conditions of constant temperature, this example exhibits quasi-periodic 398 behavior. This conclusion is supported by the Poincaré section in Figure 9c, which shows a 399 closed curve, and the spectral analysis of the time series (Fig. 9d), which yields a relatively 400 small number of peaks.

401 Not only is irregular behavior observed when the leaf temperature is forced, but it is 402 also observed when the light is forced, for certain ranges of temperature. For example, 
403 forcing the light in the manner described in Section 4 results in a possible chaotic solution 404 when the temperature is held constant at $T_{l}=285 K$ (Fig. 9e, f), which is supported by 405 the Poincaré section which shows two disjoint curves (Fig. 9g) reminiscent of the Poincaré 406 section obtained from other chaotic attractors such as the Lorenz attractor [11]. The spectral 407 analysis, which shows a less distinctive pattern of frequencies, also supports this possibility 408 (Fig. 9h). It is interesting to note that, while the development of chaos disrupts the plants' 409 functioning significantly, it still undergoes an average of one oscillation every 24 hours (Fig. $4109 \mathrm{e})$.

\section{5. Discussion and Conclusion}

412 The model presented in [2], which for the first time fully combines a CAM carbon assimilation 413 model [15] with a model for the circadian dynamical system, allows a realistic coupling of 414 CAM photosynthesis to changing environmental conditions [2], showing how the endogenous 415 circadian rhythm of CAM can be entrained by environmental forcing and how different types 416 of forcing can lead to qualitatively different responses in the temporal behavior and carbon 417 assimilation of CAM plants. Under certain circumstances, the nonlinearities and feedbacks 418 between water status, energy input, and the circadian rhythm of the model give rise to quasi419 periodic or chaotic behavior. While the observation of complex temporal dynamics, including 420 chaos, in CAM plants is not new [34, 23], the results given here provide a more detailed and 421 realistic analysis of the conditions under which complex behavior can be expected to occur 422 and what effect this type of behavior may have on plant productivity. They are also the first 423 to show quasi-periodicity in a model of CAM plants.

424 Although periodic forcing of the Liénard's equation has been mathematically explored 425 in the literature $[50,10]$, it has typically been done through the additive forcing provided by 426 the introduction of a fourth term, $p(t)$, to the general equation, i.e.,

$$
\frac{d^{2}}{d t^{2}}(x)+f(x) \frac{d}{d t} x+g(x)=p(t)
$$

427 where $p(t)$ is a periodic function of a given frequency. In this general case, it has been 
428 found that there exist stable and unstable periodic solutions with the resulting period equal 429 to that of the forcing term [50]. On the other hand, the periodic forcing of our system 430 (see Eq. 5) appears in a more complex form through the parameters of leaf temperature $431 T_{l}$, which appears in the terms $f(x)$ and $g(x)$, and solar radiation $\phi$, which appears in $432 g(x)$. Consequently this forcing introduces a more complex response which results in the 433 observed quasi-periodic and chaotic behaviors (see Fig. 9). These responses arise due to 434 nonlinearities of the model, and as would be expected, changes in the leaf temperature in 435 three key nonlinear terms (the malic acid equilibrium expression $M_{E}\left(z, T_{l}\right)$ and the carbon 436 fluxes $A_{s v}\left(M, z, T_{l}, \psi_{l}, \phi\right)$ and $\left.A_{v c}\left(M, z, T_{l}, \phi\right)\right)$, and changes in the solar radiation in the one 437 key nonlinear term (the carbon flux $\left.A_{v c}\left(M, z, T_{l}, \phi\right)\right)$ both result in possible chaos and/or 438 quasiperiodicity. Despite these key differences, the forced system explored in this paper takes 439 on the period of the forcing term(s) as in the general case of Eq. 12 [50]. Thus the model 440 is always synchronized with the daily diurnal/nocturnal cycle, as would be expected for a 441 relaxation oscillation.

442 Quasi-periodic and chaotic behaviors are not uncommon in biology and have been pre443 viously observed in the circadian rhythms of both plants and animals [25, 23]. In certain 444 situations such behaviors may, in fact, function to increase robustness under varied en445 vironmental conditions by allowing for greater flexibility, as has been proposed by Lloyd 446 (1997). Indeed, our model results suggest that CAM plants are able to maintain a relatively 447 high level of carbon assimilation under conditions which cause a chaotic or quasi-periodic 448 response. While the periodic forcing of light and/or temperature encourages irregular re449 sponses, it also extends the temperature range over which carbon assimilation will occur via 450 the CAM pathway. Under constant environmental conditions, malic acid will diffuse from 451 the vacuole and the CAM system will stall at temperatures above about $300 \mathrm{~K}$. Under forced 452 environmental conditions, while the behavior may become more erratic, the plant is able to 453 continue assimilation via CAM for the entire temperature range of 283 to $302 \mathrm{~K}$.

454 The emergence of either quasi-periodicity or chaos often causes changes in the main 455 frequency of oscillation, but it does allow CAM photosynthesis to continue with some reg456 ularity, with the time between peaks in the circadian rhythm order ranging from about 24 
457 to 30 hours. Overall, the transitions to quasi-periodicity or chaos, which are reversible with 458 small changes in light or temperature [18], appear relatively infrequently under typical en459 vironmental conditions and still allow CAM plants to maintain an adequate level of carbon 460 assimilation.

461 In summary, the results presented here demonstrate the sensitivity of CAM plants to 462 external forcing and show how certain combinations of environmental conditions can lead 463 to stable fixed points, rhythmic oscillations, and possible chaotic behavior. Like earlier 464 models of CAM, the model explored here has a dependency on leaf temperature, which is a 465 controlling parameter in the stability of the circadian oscillations of $z$ and $M$. It also shows 466 a dependency on water availability, with more negative leaf potentials inhibiting carbon 467 assimilation and less negative leaf potentials increasing assimilation. A CAM cycle period of 46824 hours is found under constant environmental conditions. However, when an acclimation 469 factor adjusts the carbon fluxes for the average temperature found over multiple days, the 470 period of CAM measured experimentally $[56,19,4]$ matches the model period for a range of 471 temperatures. This acclimation factor suggestions that studies of CAM productivity should 472 account for the change in the average daily temperature that occurs over the course of a 473 growing season. While changing the magnitude of a constant level of light has little to no 474 effect on the circadian rhythm of the model, periodic forcings of light and temperature may, 475 in some cases, lead to quasi-periodicity and/or chaos, but more importantly such forcings 476 extend the range of temperature under which assimilation is expected to occur. In addition, 477 the predicted behavior of this CAM model as a function of temperature agrees well with 478 experimental studies.

479 While moisture conditions in this work were considered indirectly through the leaf poten480 tial, it would be interesting to also couple this CAM model with the soil-plant-atmosphere 481 continuum model component of [2] and introduce forcing with stochastic rainfall to inves482 tigate the hardiness of CAM plants in environments with varying water availabilities. This 483 would allow an exploration of carbon vs. water starvation in CAM plants and estimates of 484 CAM productivity for particular species in a given area. 


\section{Acknowledgements}

486 This work was partially funded through the Agriculture and Food Research Initiative of 487 the USDA National Institute of Food and Agriculture (2011-67003-30222); the National Sci488 ence Foundation through grants CBET-1033467, EAR-1331846, FESD-1338694, and EAR489 1316258; and by the U.S. Department of Energy (DOE) through the office of Biological and 490 Environmental Research (BER) Terrestrial Carbon Processes Program (DE-SC0006967). We 491 thank Prof. Paolo Inglese of the Universitá degli Studi di Palermo for his useful suggestions 492 and comments.

\section{Appendix}

494 In the following appendices, we provide brief descriptions of the model components first 495 presented in [2].

496 A. Mass carbon balance

497 As per [2], the net influx of $\mathrm{CO}_{2}$ into the plant cytosol from the stomata, $A_{n}$, is assumed to 498 be in equilibrium with the outgoing $\mathrm{CO}_{2}$ fluxes, $A_{s c}$ to the Calvin cycle and $A_{s v}$ to the cell 499 vacuole, i.e.,

$$
A_{n}=A_{s c}+A_{s v}
$$

500 The dark respiration flux, $R_{d}$, from the mitochondria, consists of two components, $R_{d c}$, the 501 flux emitted to the Calvin cycle, and $R_{d v}$, the flux to the cell vacuole, i.e.,

$$
R_{d}=R_{d c}+R_{d v}
$$

502 At the Calvin cycle site, the carbon flux, $A_{c}$, is supplied by the dark respiration as $R_{d c}$, by 503 the stomatal cavity as $A_{s c}$, or by the cell vacuole as $A_{v c}$, i.e.,

$$
A_{c}=A_{s c}+A_{v c}+R_{d c}
$$


504 The $\mathrm{CO}_{2}$ influx $A_{n}$ is supplied from the atmosphere as steady-state Fickian diffusion with 505 diffusion coefficient $g_{m s, C O_{2}}=\frac{g_{m, C O_{2}} g_{s, C O_{2}}}{\left(g_{m, C O}+g_{s, C O_{2}}\right)}$ (the series of the mesophyll and stomatal con506 ductance) as

$$
A_{n}=g_{m s, C O}\left(c_{s}-c_{i}\right)
$$

507 where $c_{s}$ is the atmospheric concentration of $\mathrm{CO}_{2}$ and $c_{i}$ is the cytosol concentration of $\mathrm{CO}_{2}$. 508 From Equation A.4, $g_{m s, C O_{2}}$ can be expressed as

$$
g_{m s, C O_{2}}=\frac{A_{n}}{c_{s}(1-R)}
$$

509 where $R$ is the ratio of mesophyll to atmospheric $\mathrm{CO}_{2}$ concentration [41, 42]. Following [30], $510 R$ is expressed as a function of vapor pressure deficit, $D$, as

$$
R=1-\frac{1}{a_{1}}\left(1+\frac{D}{D_{x}}\right)
$$

511 with empirical constants $a_{1}$ and $D_{x}$.

512 B. Carbon assimilation model

513 As per [2], the net photosynthetic demand for $\mathrm{CO}_{2}$ from the cell vacuole, $A_{v c}$, is modeled 514 after Farquhar et. al. [15] and is expressed as a function of solar radiation, $\phi\left(\mathrm{W} / \mathrm{m}^{2}\right)$, 515 mesophyll $\mathrm{CO}_{2}$ concentration, $c_{c}(\mathrm{ppm})$, and the leaf temperature $T_{l}(\mathrm{~K})$, i.e.,

$$
A_{\phi, c_{i}, T_{l}}=f\left(\phi, c_{c}, T_{l}\right)=\min \left(A_{c}, A_{Q}\right),
$$

516 where $A_{c}$, the Rubisco-limited photosynthesis, is modeled as

$$
A_{C}=V_{c, \max }\left(T_{l}\right) \frac{c_{c}-\Gamma^{*}}{c_{c}+K_{c}\left(1+o_{i} / K_{o}\right)}
$$

517 and $A_{Q}$, the electron transport-limited photosynthesis, is modeled as

$$
A_{Q}=\frac{J\left(c_{c}-\Gamma^{*}\right)}{4\left(c_{c}+2 \Gamma^{*}\right)}
$$


518 where $V_{\max }\left(T_{l}\right)$ is the maximum carboxylation rate, $K_{c}$ and $K_{o}$ are the Michaelis-Menten 519 coefficients for $\mathrm{CO}_{2}$ and $\mathrm{O}_{2}$, respectively, and $\Gamma^{*}$ is the $\mathrm{CO}_{2}$ compensation point. For the 520 Phase III flux $A_{v c}$ (Eq. 4), notice that the C3 demand $A_{\phi, c_{c}, T_{l}}$ is no longer driven by the 521 lower concentration $c_{i}$ of Phases II and IV but instead is driven by the larger concentration $c_{c}$ 522 because of the increase in the mesophyll cytosol $\mathrm{CO}_{2}$ from the Phase III decarboxylation of 523 malic acid $\left[2\right.$, p. 117]. In $A_{Q}, J$ is the electron transport rate and is equal to $\min \left(J_{\max }\left(T_{l}\right), Q\right)$ 524 where $Q$ is the amount of solar radiation $\phi$ (in $\left.W / m^{2}\right)$ converted to mol electrons $\left(M^{-2} s^{-1}\right)$. $525 Q$ is given by

$$
Q(\phi)=\frac{\phi \lambda}{N_{a} h c} \times 0.5 \times \kappa_{2}
$$

526 where 50 percent of the incoming radiation is considered photosynthetically active radiation 527 (PAR) [21], $\lambda$ is the average wavelength (m) for PAR (assumed to be $550 \mathrm{~nm}$ ), $h$ is Planck's 528 constant $(\mathrm{Js}), c$ is the speed of light $(\mathrm{m} / \mathrm{s}), N_{a}$ is Avogadro's constant $\left(\mathrm{mol}^{-1}\right)$, and $\kappa_{2}$ is 529 the quantum yield of photosynthesis in mol $\mathrm{CO}_{2} \mathrm{~mol}^{-1}$ photons (assumed to be $0.3[31,2]$ ). $530 J_{\max }\left(T_{l}\right)$ is given by

$$
J_{\text {max }}\left(T_{l}\right)=J_{\max 0} \frac{\exp \left[\frac{H_{v J}}{R T_{0}}\left(1-\frac{T_{0}}{T_{l}}\right)\right]}{1+\exp \left(\frac{S_{v J} T_{l}-H_{d J}}{R T_{l}}\right)} .
$$

531 The maximum carboxylation rate, $V_{c, \max }$, and the $\mathrm{CO}_{2}$ compensation point, $\Gamma^{*}$, are given 532 by

$$
V_{c, \max }\left(T_{l}\right)=V_{c, \max 0} \frac{\exp \left[\frac{H_{v V}}{R T_{0}}\left(1-\frac{T_{0}}{T_{l}}\right)\right]}{1+\exp \left(\frac{S_{v V} T_{l}-H_{d V}}{R T_{l}}\right)}
$$

533 and

$$
\Gamma^{*}\left(T_{l}\right)=\gamma_{0}\left[1+\gamma_{1}\left(T_{l}-T_{0}\right)+\gamma_{2}\left(T_{l}-T_{0}\right)^{2}\right]
$$

534 The temperature dependence of the Michaelis-Menten constants $K_{c}$ and $K_{o}$ is described by 535 a modified Arrhenius equation, i.e.,

$$
K_{x}\left(T_{l}\right)=K_{x 0} \exp \left[\frac{H_{K x}}{R T_{0}}\left(1-\frac{T_{0}}{T_{l}}\right)\right],
$$

536 where $x$ stands for either $c$ or $o$. Parameter values can be found in Table 3. 
537 C. Dark Respiration $\mathrm{CO}_{2}$ flux

538 A modified Arrhenius equation also describes the temperature dependence of the flux of 539 carbon from dark respiration [26], i.e.,

$$
R_{d}\left(T_{l}\right)=R_{d 0} \exp \left[\frac{H_{R d}}{R T_{0}}\left(1-\frac{T_{0}}{T_{l}}\right)\right]
$$

540 This flux of carbon is assumed to be redirected to either the Calvin cycle, $R_{d c}$, or to the 541 cell vacuole $R_{d v}$. When solar radiation, $\phi$, is non-zero, almost all dark respiration $\mathrm{CO}_{2}$ is 542 directed to the Calvin cycle such that

$$
R_{d c}\left(T_{l}, \phi\right)=R_{d}\left(T_{l}\right)(1-\exp (-\phi))
$$

543 When solar radiation is absent, i.e., $\phi=0$, we assume that all dark respiration $\mathrm{CO}_{2}$ is 544 converted to malic acid and transported to the cell vacuole, i.e.,

$$
R_{d v}\left(T_{l}, \phi\right)=R_{d}\left(T_{l}\right) \exp (-\phi)
$$

545 D. Effect of leaf water potential

546 We assume that the net $\mathrm{C}$ uptake for malic acid storage is negatively impacted at leaf water 547 potentials below $\psi_{l 1}$ and linearly decreases to zero at $\psi_{l 0}$ [13], i.e.,

$$
f_{\psi l}\left(\psi_{l}\right)=\left\{\begin{array}{lr}
0 & \psi_{l}<\psi_{l_{0}} \\
\frac{\psi_{l}-\psi_{l_{0}}}{\psi_{l_{1}}-\psi_{l_{0}}} & \psi_{l_{0}} \leq \psi_{l} \leq \psi_{l_{1}} \\
1 & \psi_{l_{1}}<\psi_{l} \leq 0
\end{array}\right.
$$

548 E. Effect of temperature on nocturnal carbon uptake

549 Based on experimental data $[41,37,43,59]$, we assume that the temperature dependence on 550 the net carbon uptake of Phase I has a parabolic form centered at an optimum temperature, 
551 i.e.,

$$
A_{s v, \max }\left(T_{l}\right)=A_{s v, \max 0}\left[1-k\left(T_{l}-T_{o p t}\right)^{2}\right]
$$

552 where $T_{l}$ is the leaf temperature, $T_{\text {opt }}$ is the optimal leaf temperature, $A_{s v, \max 0}$ is the optimum

553 rate of carbon fixation as malic acid, and $k$ is a scale parameter (see [2]).

554 F. Equilibrium value of malic acid

555 The cubic function $M_{E}\left(z, T_{l}\right)$ represents the equilibrium concentration of vacuolar malic acid 556 for the current circadian order $z$,

$$
M_{E}\left(z, T_{l}\right)=M_{\max }\left[\left(\frac{T_{H}-T_{l}}{T_{H}-T_{L}}+1\right) c_{1}[\beta(z-\mu)]^{3}-\frac{T_{H}-T_{l}}{T_{H}-T_{L}}\left[\beta(z-\mu)-c_{2}\right]\right],
$$

557 where $T_{l}$ is the leaf temperature $(\mathrm{K})$, and $c_{1}, c_{2}, \beta$ and $\mu$ are constants which function 558 to position the curve [2]. $T_{H}=302.15 \mathrm{~K}$ and $T_{L}=283.15 \mathrm{~K}$ represent the bounds of the 559 average temperature range over which the circadian rhythm of CAM has been observed $560[56,34,41,12,9,19,20]$.

561 G. Order function

562 The order function $f_{O}(z)$ represents the activity of the enzyme PEPC, the vacuolar $\mathrm{H}+-$ 563 ATPase and/or H+-PPase pump for malic acid, and the decarboxylation enzymes for malic 564 acid, which control the creation and release of malic acid. We include the functional depen565 dency on $z$ as a switch represented by

$$
f_{O}(z)=\exp \left[-\left(\frac{z}{\mu}\right)^{c_{3}}\right]
$$

566 where $\mu$ is the position parameter and $c_{3}$ controls the slope of $f_{O}(z)[2] . f_{O}(z)$ is a sigmoidal 567 curve comprised between 0 and 1, of the type often used as a vulnerability function in plant 568 physiology $[52,54]$. 
569 H. Storage of malic acid

570 The maximum concentration of malic acid that the system is capable of storing is given by

$$
M_{S}\left(T_{l}\right)=M_{\max }\left[\frac{T_{H}-T_{l}}{T_{H}-T_{L}}\left(1-\alpha_{2}\right)+\alpha_{2}\right]
$$

571 where $T_{H}$ and $T_{L}$ are the temperature range values of the circadian rhythm, $T_{l}$ is the leaf 572 temperature, $M_{\max }$ is the maximum malic acid concentration at $T_{L}$, and $\alpha_{2}$ is a parameter 573 between 0 and 1 . At small values of $\alpha_{2}$, the disparity between the maximum equilibrium 574 value, $M_{E, \max }\left(z, T_{l}\right)$ and the storage limitation $M_{S}\left(T_{l}\right)$ is small, and a smaller half reaction 575 constant of $\alpha_{2} M_{S}\left(T_{l}\right)$ is required to temper the malic acid flux to the vacuole near the 576 equilibrium concentration, $M_{E}\left(z, T_{l}\right)$. When $\alpha_{2}$ is large, the disparity between $M_{E, \max }\left(z, T_{l}\right)$ 577 and $M_{S}\left(T_{l}\right)$ increases, and a larger half reaction constant is required to temper the malic 578 acid flux to the vacuole near the equilibrium concentration. 
Table 1: General model parameters

\begin{tabular}{ccll}
\hline \hline Parameter & Value & Units & Description \\
\hline$a_{1}$ & $6.4^{\mathrm{a}}$ & & Eq. A.6 \\
$D_{x}$ & $0.0068^{\mathrm{a}}$ & $\mathrm{kg} \mathrm{kg}^{-1}$ & Eq. A.6 \\
$\frac{g_{m, C O_{2}}}{g_{s, C O_{2}}}$ & $1^{\mathrm{b}}$ & & Mesophyll conductance (unit leaf area) \\
$p_{a}$ & $1.013 \cdot 10^{5}$ & $\mathrm{~Pa}$ & Air pressure \\
$\lambda_{w}$ & $2.5 \cdot 10^{6}$ & $\mathrm{~J} \mathrm{~kg}^{-1}$ & Latent heat of water vaporization \\
$\rho$ & 1.2 & $\mathrm{~kg} \mathrm{~m}^{-3}$ & Air density \\
$\psi_{1_{1}}$ & $-0.5^{\mathrm{c}}$ & $\mathrm{MPa}$ & Eq. D.1 \\
$\psi_{l_{0}}$ & $-3^{\mathrm{c}}$ & $\mathrm{MPa}$ & Eq. D.1 \\
$T_{H}$ & $302.15^{\mathrm{d}}$ & $\mathrm{K}$ & Eq. F.1 \\
$T_{L}$ & $283.15^{\mathrm{d}}$ & $\mathrm{K}$ & Eq. F.1 \\
$t_{r}$ & 95 & $\mathrm{~min}$ & Eq. 1, relaxation time \\
$\alpha_{1}$ & $1 / 100$ & & Eq. $4,0 \leq \alpha_{1} \leq 1$ \\
$\alpha_{2}$ & $1 / 7$ & & Eq. H.1, $0 \leq \alpha_{2} \leq 1$ \\
$k$ & $0.003^{\mathrm{e}}$ & & Eq. E. 1 \\
$T_{o p t}$ & $288.65^{\mathrm{e}}$ & $\mathrm{K}$ & Eq. E. 1 \\
$c_{a}$ & 350 & $\mathrm{ppm}$ & Atmospheric $\mathrm{CO}_{2}$ concentration \\
$c_{c}$ & 500 & $\mathrm{ppm}$ & Cytoplasm $\mathrm{CO}_{2}$ concentration \\
$b_{1}$ & 8.3 & & Eq. 9 \\
$b_{2}$ & $1 / 7$ & & Eq. 9 \\
\hline
\end{tabular}

a Average value for $\mathrm{C}_{3}$ from Table 2 (Lohammer column) in [26].

b Based on ratio for an arid environment [16].

c Based on the data of [28] and [38].

${ }^{d}$ Based on data discussed in Appendix F.

e Best fit to the nocturnal carbon uptake data of [41]. 
Table 2: Model parameters specific to Agave tequilana

\begin{tabular}{|c|c|c|c|}
\hline Parameter & Value & Units & Description \\
\hline \multicolumn{4}{|c|}{ Plant Parameters } \\
\hline$L A I$ & $6^{\mathrm{a}}$ & & Leaf Area Index \\
\hline$L_{M}$ & $2.7^{\mathrm{b}}$ & $\mathrm{mm}$ & Malic acid stor. vol. to leaf surf. ratio \\
\hline$V_{c, \max 0}$ & $16.5^{\mathrm{c}}$ & $\mu \mathrm{mol}_{\mathrm{CO}_{2}} \mathrm{~m}^{-2} \mathrm{~s}^{-1}$ & Eq. B.6 \\
\hline$M_{\max }$ & $126^{\mathrm{c}}$ & $\mathrm{mol} \mathrm{m} \mathrm{m}^{-3}$ & Maximum malic acid concentration \\
\hline$A_{s v, \max 0}$ & $11.25^{\mathrm{c}}$ & $\mu \mathrm{mol} \mathrm{m}{ }^{-2} \mathrm{~s}^{-1}$ & Max. rate for malic acid synthesis \\
\hline \multicolumn{4}{|c|}{ Model Initial Conditions } \\
\hline$z$ & 0.5 & & Circadian order \\
\hline$M$ & 0 & $\mathrm{~mol} \mathrm{~m}^{-3}$ & Malic acid concentration \\
\hline \multicolumn{4}{|c|}{$\begin{array}{l}\text { a }[37,40] \text { on a total leaf area basis (both sides). } \\
\text { b Based on chlorenchyma thickness (one leaf side) of } 3 \mathrm{~mm}[37, \text { p. } 40] \text { adjusted by at } \\
\text { intracellular air space of } 10 \text {-percent }[37, \mathrm{p} .41] . \\
{ }^{\mathrm{c}} \text { Based on the carbon flux data of }[44] \text { and }[24] \text {. }\end{array}$} \\
\hline
\end{tabular}


Table 3: Parameters for photosynthesis model

\begin{tabular}{ccll}
\hline \hline Parameter & Value & Units & Description \\
\hline$H_{K c}$ & $59430^{\mathrm{a}}$ & $\mathrm{J} \mathrm{mol}^{-1}$ & Activation energy for $K_{c}$ \\
$H_{K o}$ & $36000^{\mathrm{a}}$ & $\mathrm{J} \mathrm{mol}^{-1}$ & Activation energy for $K_{o}$ \\
$H_{R d}$ & $53000^{\mathrm{a}}$ & $\mathrm{J} \mathrm{mol}^{-1}$ & Activation energy for $R_{d}$ \\
$R_{d 0}$ & $0.32^{\mathrm{a}}$ & $\mu \mathrm{mol} \mathrm{mol}^{-1}$ & Dark respiration at $T_{0}$ \\
$H_{v V}$ & $72000^{\mathrm{c}}$ & $\mathrm{J} \mathrm{mol}^{-1}$ & Activation energy for $V_{c, \text { max }}$ \\
$H_{d V}$ & $200000^{\mathrm{c}}$ & $\mathrm{J} \mathrm{mol}^{-1}$ & Deactivation energy for $V_{c, \text { max }}$ \\
$J_{\text {max } 0}$ & $2 V_{c, \text { max0 }} \mathrm{b}$ & $\mathrm{J} \mathrm{mol}^{-1}$ & Eq. B.5 (see Table 2) \\
$H_{v J}$ & $50000^{\mathrm{c}}$ & $\mathrm{J} \mathrm{mol}^{-1}$ & Activation energy for $J_{\max }$ \\
$H_{d J}$ & $200000^{\mathrm{c}}$ & $\mathrm{J} \mathrm{mol}^{-1}$ & Deactivation energy for $J_{\text {max }}$ \\
$K_{c 0}$ & $302^{\mathrm{a}}$ & $\mu \mathrm{mol} \mathrm{mol}^{-1}$ & Michaelis constant for CO $\mathrm{CO}_{2}$ at $T_{0}$ \\
$K_{o 0}$ & $256^{\mathrm{a}}$ & $\mathrm{mmol} \mathrm{mol}^{-1}$ & Michaelis constant for $\mathrm{O}_{2}$ at $T_{0}$ \\
$o_{i}$ & $0.209^{\mathrm{a}}$ & $\mathrm{mol} \mathrm{mol}^{-1}$ & Oxygen concentration \\
$R$ & 8.31 & $\mathrm{~J} \mathrm{~mol}^{-1} \mathrm{~K}^{-1}$ & Universal Gas constant \\
$S_{v V}$ & $649^{\mathrm{c}}$ & $\mathrm{J} \mathrm{mol}^{-1}$ & Entropy term for carboxylation \\
$S_{v J}$ & $646^{\mathrm{c}}$ & $\mathrm{J} \mathrm{mol}^{-1}$ & Entropy term for electron transport \\
$T_{o}$ & $293.2^{\mathrm{a}}$ & $\mathrm{K}^{\mathrm{a}}$ & Reference temperature \\
$\gamma_{o}$ & $34.6^{\mathrm{a}}$ & $\mu \mathrm{mol} \mathrm{mol}^{-1}$ & CO ${ }_{2}$ compensation point at $T_{0}$ \\
$\gamma_{1}$ & $0.0451^{\mathrm{a}}$ & $\mathrm{K}^{-1}$ & Eq. B.7 \\
$\gamma_{2}$ & $0.000347^{\mathrm{a}}$ & $\mathrm{K}^{-2}$ & Eq. B.7 \\
\hline
\end{tabular}

a Value for $\mathrm{C}_{3}$ per [26].

${ }^{\text {b }}$ On average $J_{\max 0}$ is twice $V_{c, \max 0}$, see [22].

${ }^{c}$ Average $\mathrm{C}_{3}$ value per [22].

Table 4: Circadian oscillator constants

\begin{tabular}{cc}
\hline \hline Constant $^{\mathrm{a}}$ & Value $^{\mathrm{b}}$ \\
\hline$c_{1}$ & 0.365 \\
$c_{2}$ & 0.55 \\
$c_{3}$ & 10 \\
$\mu$ & 0.5 \\
$\beta$ & 2.764 \\
\hline
\end{tabular}

${ }^{\mathrm{a}}$ see Appendices $\mathrm{F}$ and $\mathrm{G}$.

b Based on [2]. 
Figure 1: Carbon assimilation model schematic. The circadian order variable, $z$, and malic acid concentration, $M$, serve as a control variables for the carbon fluxes (arrows).

Figure 2: Nullclines $d z / d t=0$, shown for different leaf temperatures $\left(T_{L}=283.15 \mathrm{~K}\right.$ to $\left.T_{H}=302.15 \mathrm{~K}\right)$, and $d M / d t=0$, shown for different leaf potentials $\left(\psi_{l 2}=-2.95 \mathrm{MPa}\right.$ to $\left.\psi_{l 1}=-0.5 \mathrm{MPa}\right)$.

Figure 3: Panels a) and b) show phase diagrams of the circadian system for different relaxation time constants, $t_{r}$, when leaf potential $\psi_{l}=-0.5 \mathrm{MPa}$ and leaf temperature $T_{l}=293.15 \mathrm{~K}$. Panel c) shows effects of leaf temperature, $T_{l}$, when $\psi_{l}=-0.5 \mathrm{MPa}$. Panel d) shows effects of leaf potential, $\psi_{l}$, when $T_{l}=290 \mathrm{~K}$. Panels e) and f) display vector fields and nullclines for specific phase portraits in panels c) and d). $t_{r}=95$ minutes unless otherwise noted.

Figure 4: Panels (a-c) show the period length, bifurcation diagram, and carbon assimilation, respectively, under various leaf temperatures, and panels (d-f) show the same quantities under various leaf potentials. In (a-c), leaf potential is held constant at $-0.5 \mathrm{MPa}$, while in (d-f), leaf temperature is held constant at 293.15 K. In all cases, $t_{r}=95$ minutes.

Figure 5: Period length and comparison to experimental data, where circles represent the data from [18, 4] and squares represent the data from [56]. The carbon fluxes are adjusted by an acclimation factor of $f_{a}$ (see Eq. 9). The leaf potential is held constant at $-0.5 \mathrm{MPa}, t_{r}=95$ minutes, $T_{L}=278 \mathrm{~K}$, and $T_{H}=304 \mathrm{~K}$.

Figure 6: Bifurcation diagrams and carbon assimilation for conditions of forced temperature. In (a) and (b) the amplitude of forcing is varied with $T_{l, a v}=300 \mathrm{~K}$. In $(\mathrm{c}-\mathrm{h})$ the average temperature is varied with constant amplitude of $A=1 \mathrm{~K}(\mathrm{c}, \mathrm{d}), A=5 \mathrm{~K}(\mathrm{e}, \mathrm{f})$, and $A=10 \mathrm{~K}(\mathrm{~g}, \mathrm{~h})$. Insets from panels (a) and (b) are shown in Fig. 7.

Figure 7: Details of the bifurcation diagrams: (a) is an inset from Fig. 6a and (b),(c) are insets from Fig. $6 c$.

Figure 8: Bifurcation diagrams and carbon assimilation are shown for light forced as a piecewise quadratic equation, with temperature held constant $(\mathrm{a}, \mathrm{b})$, and light forced as a piecewise quadratic equation, with temperature forced with an amplitude of $5 \mathrm{~K}(\mathrm{c}, \mathrm{d})$. In both scenarios, the maximum light intensity is $\phi_{h}=500 \mathrm{~W} / \mathrm{m}^{2}$.

Figure 9: Time series, phase portrait, Poincaré section, and Fast Fourier Transform. In (a-d) leaf temperature is forced as a sine wave, with $T_{l, a v}=298 \mathrm{~K}$ and $A=1 \mathrm{~K}$. In (e-h) irradiance is forced $\left(\phi_{h}=500\right.$ $\mathrm{W} / \mathrm{m}^{2}$ ) with $T_{l}=285 \mathrm{~K}$. 
[1] Baker GL (1996) Chaotic dynamics: an introduction. Cambridge University Press

[2] Bartlett MS, Vico G, Porporato A (2014) Coupled carbon and water fluxes in CAM photosynthesis: modeling quantification of water use efficiency and productivity. Plant and Soil 383(1-2):111-138

[3] Black C, Barry Osmond C (2005) Crassulacean acid metabolism photosynthesis: working the night shift. In: Govindjee, Beatty J, Gest H, Allen J (eds) Discoveries in Photosynthesis, Advances in Photosynthesis and Respiration, vol 20, Springer Netherlands, pp 881-893

[4] Blasius B, Beck F, Lüttge U (1997) A model for photosynthetic oscillations in Crassulacean acid metabolism (CAM). Journal of Theoretical Biology 184(3):345-351

[5] Blasius B, Beck F, Lüttge U (1998) Oscillatory model of Crassulacean acid metabolism: structural analysis and stability boundaries with a discrete hysteresis switch. Plant, Cell \& Environment 21(8):775-784

[6] Blasius B, Neff R, Beck F, Luttge U (1998) Oscillatory model of a crassulacean acid metabolis with a dynamic hysteresis switch. The Royal Society 266:93-101

[7] Brown EN, Choe Y, Luithardt H, Czeisler CA (2000) A statistical model of the human core-temperature circadian rhythm. American Journal of Physiology - Endocrinology and Metabolism 279(3):E669-E683

[8] Bruce VG (1960) Environmental entrainment of circadian rhythms. Cold Spring Harbor Symposia on Quantitative Biology 25:29-48

[9] Buchanan-Bollig IC (1984) Circadian rhythms in Kalanchoë: effects of irradiance and temperature on gas exchange and carbon metabolism. Planta 160(3):264-271

[10] Burton T, Townsend C (1968) On the generalized Liénard equation with forcing function. Journal of Differential Equations 4(4):620-633

[11] Byrne G, Gilmore R, Letellier C (2004) Distinguishing between folding and tearing mechanisms in strange attractors. Physical Review E 70(5):056,214 
605

606

607

608

609

610

611

612

613

614

615

616

617

618

619

620

621

622

623

624

625

626

627

628

629

630

631

[12] Carter P, Fewson C, Nimmo G, Nimmo H, Wilkins M (1996) Roles of circadian rhythms, light and temperature in the regulation of phosphoenolpyruvate carboxylase in crassulacean acid metabolism. In: Winter K, Smith J (eds) Crassulacean Acid Metabolism, Ecological Studies, vol 114, Springer Berlin Heidelberg, pp 46-52

[13] Daly E, Porporato A, Rodriguez-Iturbe I (2004) Coupled dynamics of photosynthesis, transpiration, and soil water balance. Part I: Upscaling from hourly to daily level. Journal of Hydrometeorology 5(3):546-558

[14] Dodd AN, Borland AM, Haslam RP, Griffiths H, Maxwell K (2002) Crassulacean acid metabolism: plastic, fantastic. Journal of Experimental Botany 53(369):569-580

[15] Farquhar G, von Caemmerer S, Berry J (1980) A biochemical model of photosynthetic CO2 assimilation in leaves of C3 species. Planta 149(1):78-90

[16] Flexas J, Ribas-Carbó M, Diaz-Espejo A, Galmes J, Medrano H (2008) Mesophyll conductance to CO2: Current knowledge and future prospects. Plant, Cell \& Environment 31(5):602-621

[17] Friemert V, Heininger D, Kluge M, Ziegler H (1988) Temperature effects on malicacid efflux from the vacuoles and on the carboxylation pathways in crassulacean-acidmetabolism plants. Planta 174(4):453-461

[18] Grams TE, Beck F, Lüttge U (1996) Generation of rhythmic and arrhythmic behaviour of crassulacean acid metabolism in Kalanchoë daigremontiana under continuous light by varying the irradiance or temperature: Measurements in vivo and model simulations. Planta 198(1):110-117

[19] Grams TEE, Borland AM, Roberts A, Griffiths H, Beck F, Lüttge U (1997) On the mechanism of reinitiation of endogenous crassulacean acid metabolism rhythm by temperature changes. Plant Physiology 113(4):pp. 1309-1317

[20] Haag-Kerwer A, Franco AC, Lüttge U (1992) The effect of temperature and light on gas exchange and acid accumulation in the C3-CAM plant Clusia minor L. Journal of Experimental Botany 43(3):345-352 
632 [21] Jones HG (1992) Plants and microclimate: a quantitative approach to environmental 633 plant physiology. Cambridge University Press

634 [22] Kattge J, Knorr W (2007) Temperature acclimation in a biochemical model of photosyn635 thesis: a reanalysis of data from 36 species. Plant, Cell \& Environment 30(9):1176-1190

636 [23] Krempaský J, Smrinová M, Ballo P (1993) Periodicity and chaos in a photosynthetic $637 \quad$ system. Photosynthesis Research 37(2):159-164

638 [24] Lee HS, Schmitt AK, Lüttge U (1989) The response of the C3-CAM tree, Clusia rosea, 639 to light and water stress II. Internal CO2 concentration and water use efficiency. Journal 640 of Experimental Botany 40(2):171-179

641 [25] Leloup JC, Goldbeter A (1999) Chaos and birhythmicity in a model for circadian os642 643 644 645

646 [27] Lhomme JP, Elguero E, Chehbouni A, Boulet G (1998) Stomatal control of transpi647 ration: Examination of Monteith's formulation of canopy resistance. Water Resources $648 \quad$ Research 34(9):2301-2308

649 [28] Linton MJ, Nobel PS (2001) Hydraulic conductivity, xylem cavitation, and water po650 tential for succulent leaves of Agave deserti and Agave tequilana. International Journal $651 \quad$ of Plant Sciences 162(4):747-754

652 [29] Lloyd D (1997) Chaos and ultradian rhythms. Biological Rhythm Research 28(1):134653 143

654 [30] Lohammar T, Larsson S, Linder S, Falk S (1980) FAST: Simulation models of gaseous 655 exchange in Scots pine. Ecological Bulletins pp 505-523 
656 [31] Long S, Postl W, Bolhár-Nordenkampf H (1993) Quantum yields for uptake of carbon 657 dioxide in C3 vascular plants of contrasting habitats and taxonomic groupings. Planta $658 \quad 189(2): 226-234$

659 [32] Lüttge U (2000) The tonoplast functioning as the master switch for circadian regulation 660 of crassulacean acid metabolism. Planta 211:761-769

661 [33] Lüttge U (2004) Ecophysiology of crassulacean acid metabolism (CAM). Annals of 662 botany $93(6): 629$

663 [34] Lüttge U, Beck F (1992) Endogenous rhythms and chaos in crassulacean acid 664 metabolism. Planta 188(1):28-38

665 [35] Lüttge U, Duarte HM (2007) Morphology, anatomy, life forms and hydraulic architec$666 \quad$ ture. In: Clusia, Springer, pp 17-30

667 [36] Nimmo G, Wilkins M, Fewson C, Nimmo H (1987) Persistent circadian rhythms 668 in the phosphorylation state of phosphoenolpyruvate carboxylase from Bryophyllum 669 fedtschenkoi leaves and in its sensitivity to inhibition by malate. Planta 170(3):408-415

670 [37] Nobel P (1988) Environmental biology of agaves and cacti. Cambridge: Cambridge $671 \quad$ University Press

672 [38] Nobel PS (1976) Water relations and photosynthesis of a desert CAM plant, agave 673 deserti. Plant Physiology 58(4):576-582

674 [39] Nobel PS (1991) Achievable productivities of certain cam plants: basis for high values 675 compared with C3 and C4 plants. New Phytologist 119(2):183-205

676 [40] Nobel PS (1994) Remarkable agaves and cacti. Oxford University Press

677 [41] Nobel PS, Hartsock TL (1978) Resistance analysis of nocturnal carbon dioxide uptake by 678 a Crassulacean acid metabolism succulent, Agave deserti. Plant Physiology 61(4):510$679 \quad 514$ 
680 [42] Nobel PS, Hartsock TL (1979) Environmental influences on open stomates of a crassu681 lacean acid metabolism plant, Agave deserti. Plant Physiology 63(1):63-66

682 [43] Nobel PS, Hartsock TL (1981) Shifts in the optimal temperature for nocturnal CO2 683 uptake caused by changes in growth temperature for cacti and agaves. Physiologia Plan$684 \quad$ tarum 53(4):523-527

685 [44] Nobel PS, Valenzuela AG (1987) Environmental responses and productivity of the CAM 686 plant, Agave tequilana. Agricultural and Forest Meteorology 39(4):319-334

687 [45] Nungesser D, Kluge M, Tolle H, Oppelt W (1984) A dynamic computer model of the 688 metabolic and regulatory processes in crassulacean acid metabolism. Planta 162(3):204$689 \quad 214$

690 [46] Osmond C (1978) Crassulacean acid metabolism: a curiosity in context. Annual Review 691 of Plant Physiology 29(1):379-414

692 [47] Owen NA, Griffiths H (2013) A system dynamics model integrating physiology and 693 biochemical regulation predicts extent of crassulacean acid metabolism (CAM) phases. $694 \quad$ New Phytologist

695 [48] Pikovsky A, Rosenblum M, Kurths J (2001) Synchronization: A universal concept in 696 nonlinear sciences. Cambridge University Press

697 [49] van der Pol B, van der Mark J (1928) The heartbeat considered as a relaxation oscilla698 tion, and an electrical model of the heart. Philosophical Magazine Series 7 6(38):763-775

699 [50] Ponzo PJ (1967) Forced oscillations of the generalized Lienard equation. SIAM Journal $700 \quad$ on Applied Mathematics 15(1):pp. 75-87

701 [51] Reicosky D, Winkelman L, Baker J, Baker D (1989) Accuracy of hourly air tempera702 tures calculated from daily minima and maxima. Agricultural and Forest Meteorology $703 \quad 46(3): 193-209$

704 [52] Sperry J, Hacke U, Oren R, Comstock J (2002) Water deficits and hydraulic limits to 705 leaf water supply. Plant, Cell \& Environment 25(2):251-263 
706 [53] Strogatz S (2001) Nonlinear dynamics and chaos: with applications to physics, biology, 707 chemistry and engineering. Perseus Books Group

708 [54] Vico G, Porporato A (2008) Modelling C3 and C4 photosynthesis under water-stressed 709 conditions. Plant and Soil 313(1-2):187-203

710 [55] Wiebe R, Virgin LN (2012) A heuristic method for identifying chaos from frequency 711 content. Chaos: An Interdisciplinary Journal of Nonlinear Science 22(1):013136

712 [56] Wilkins MB (1992) Tansley review No. 37 Circadian rhythms: their origin and control. $713 \quad$ New Phytologist 121(3):347-375

714 [57] Winter K, Garcia M, Holtum JAM (2008) On the nature of facultative and constitutive 715 CAM: environmental and developmental control of CAM expression during early growth 716 of Clusia, Kalancho, and Opuntia. Journal of Experimental Botany 59(7):1829-1840

717 [58] de Wit C (1978) Simulation of assimilation, respiration, and transpiration of crops. $718 \quad$ Simulation monographs, Wiley

719 [59] Yamori W, Hikosaka K, Way D (2014) Temperature response of photosynthesis in C3, 720 C4, and CAM plants: temperature acclimation and temperature adaptation. Photosyn$721 \quad$ thesis Research 119(1-2):101-117 
Figure 1

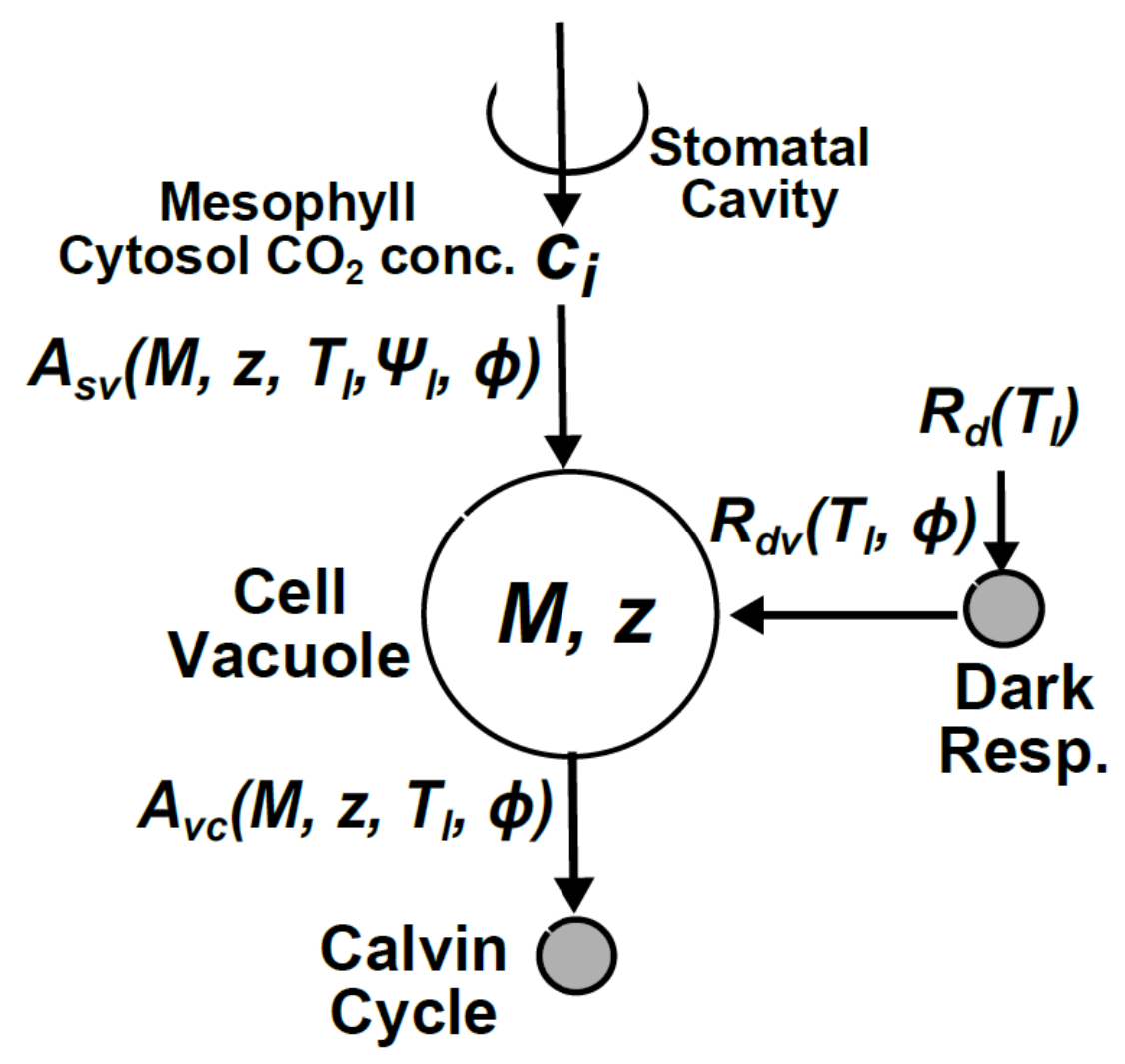


Figure 2

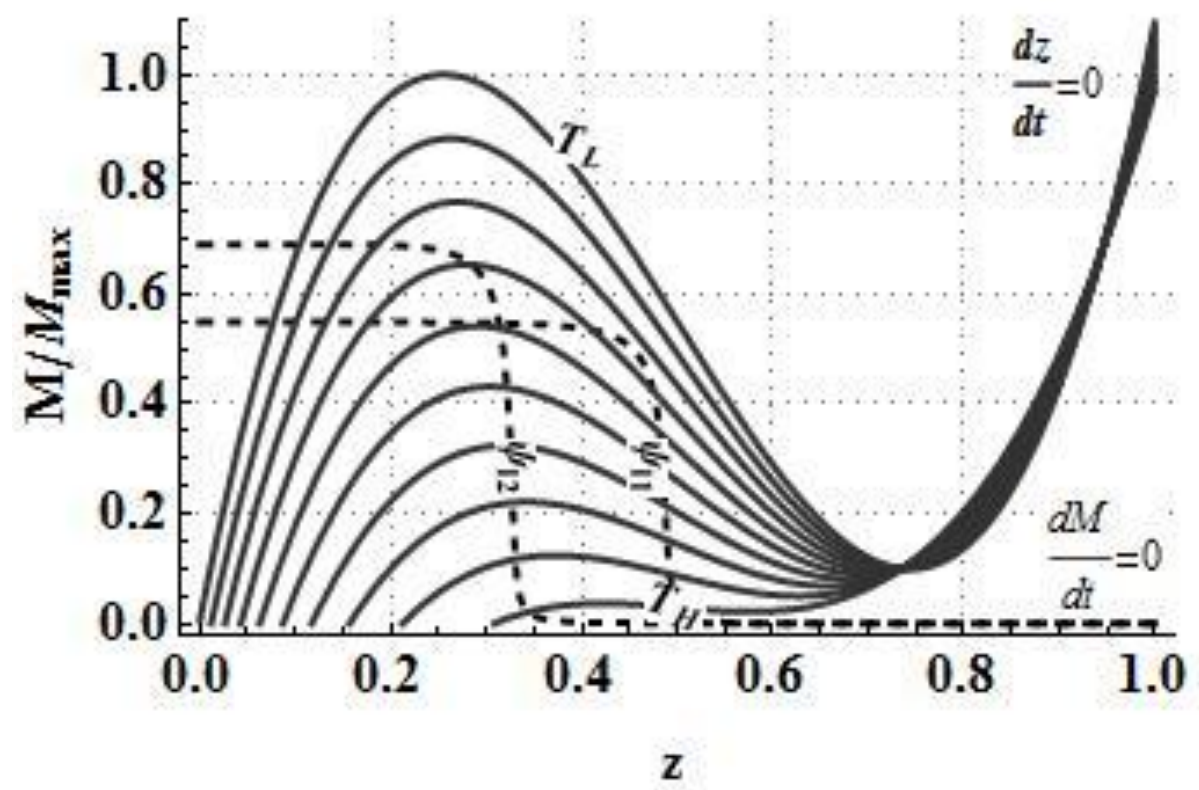




\section{Figure 3}
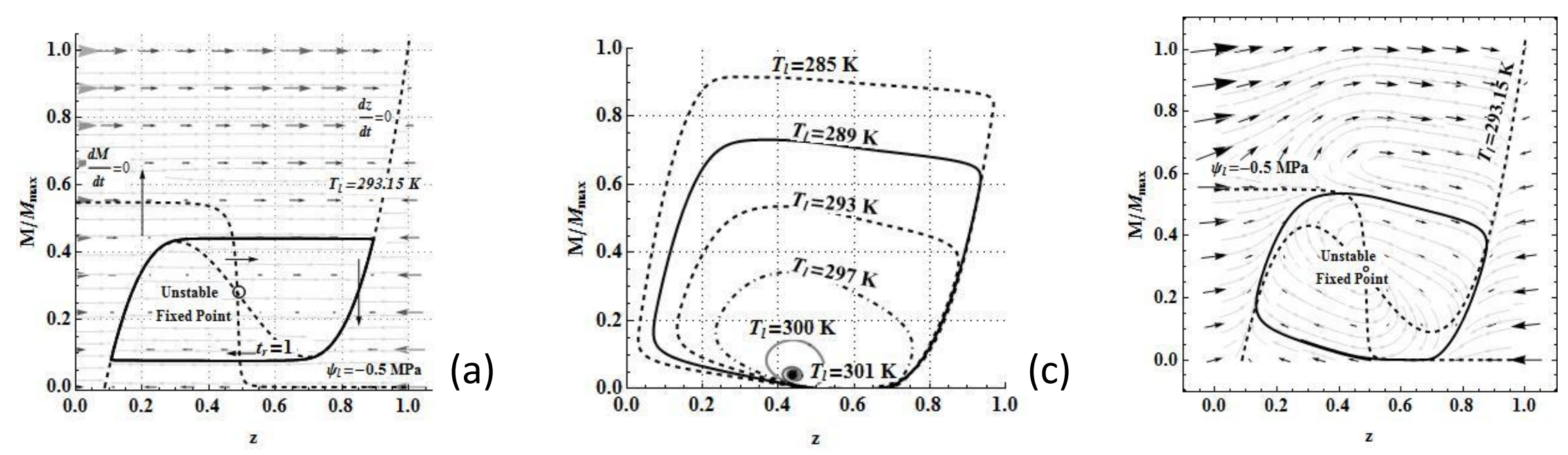

(e)

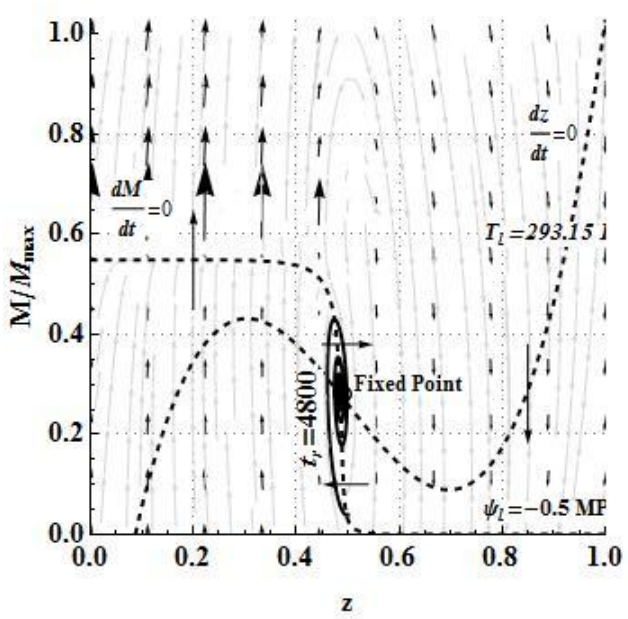

(b)
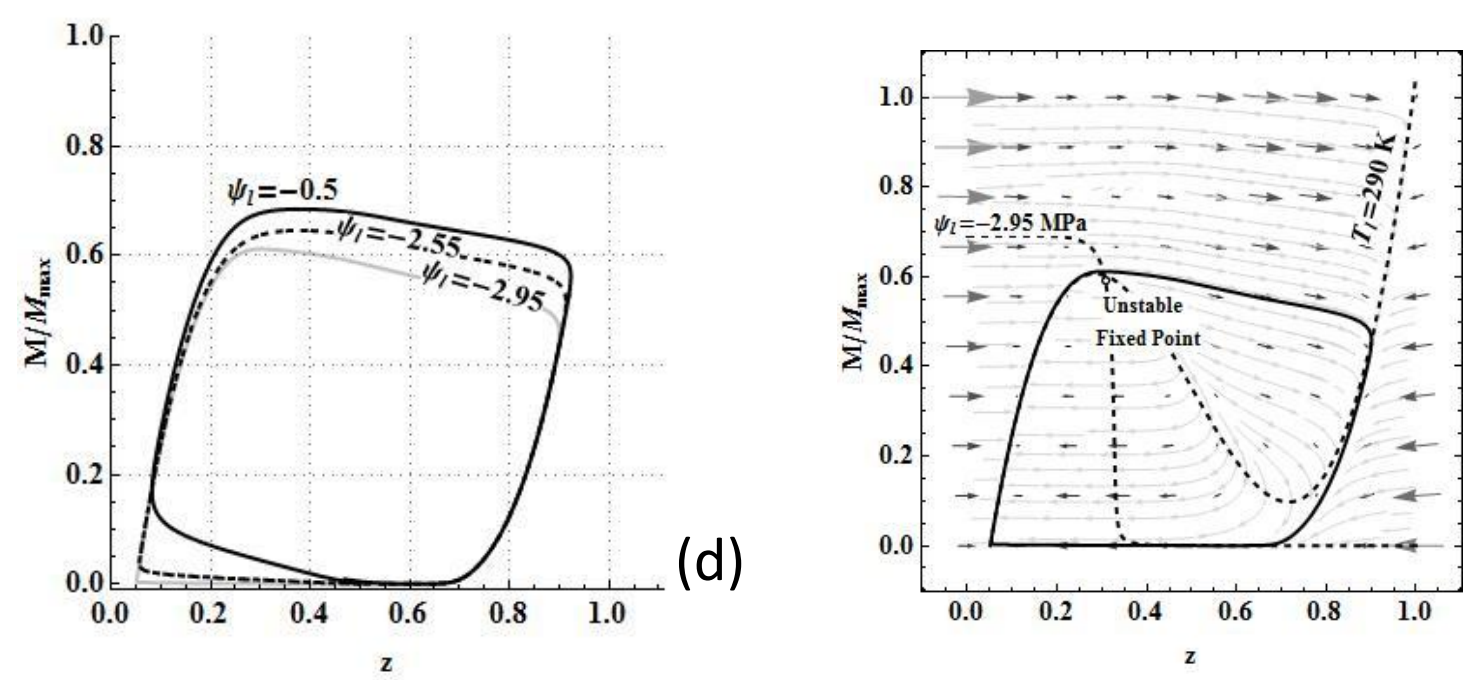
Figure 4
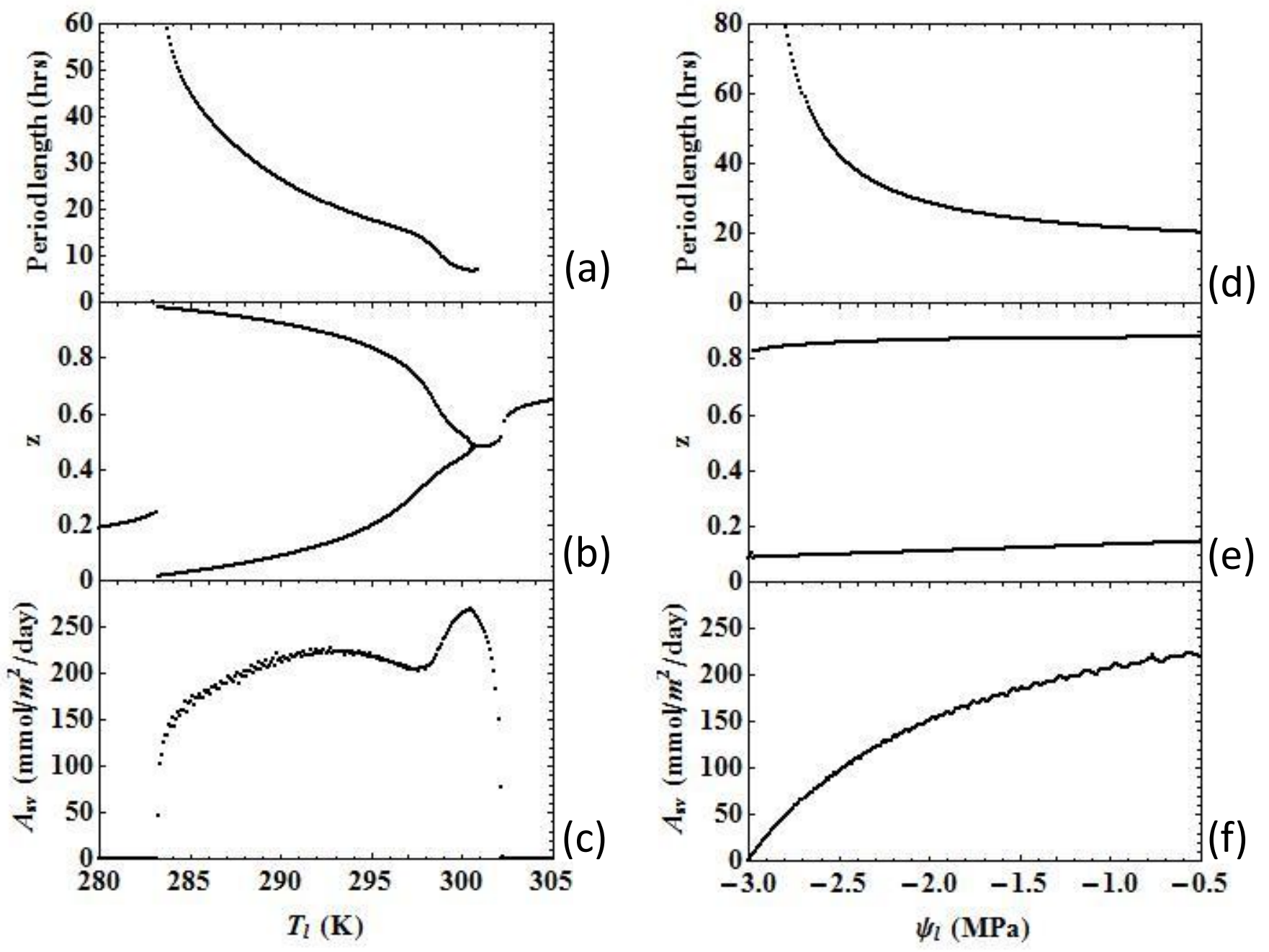
Figure 5

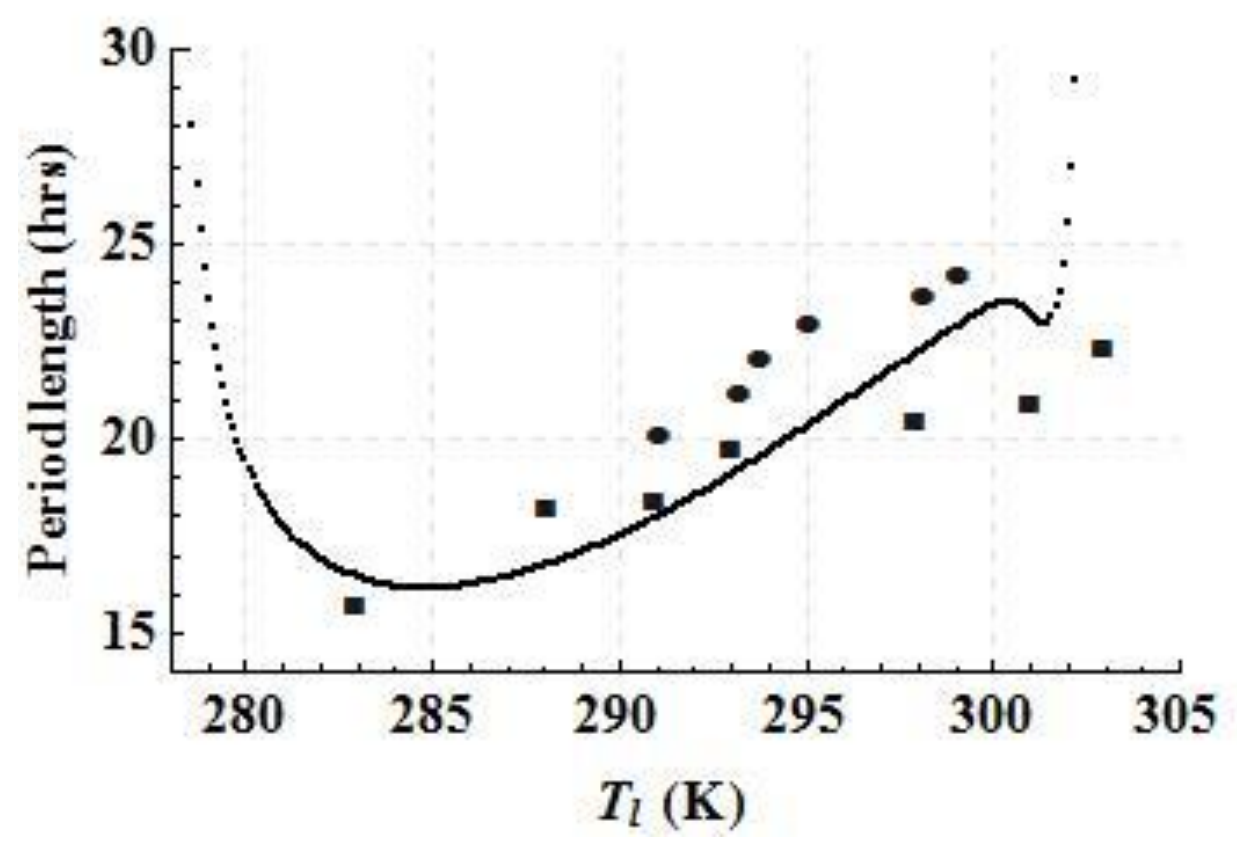




\section{Figure 6}
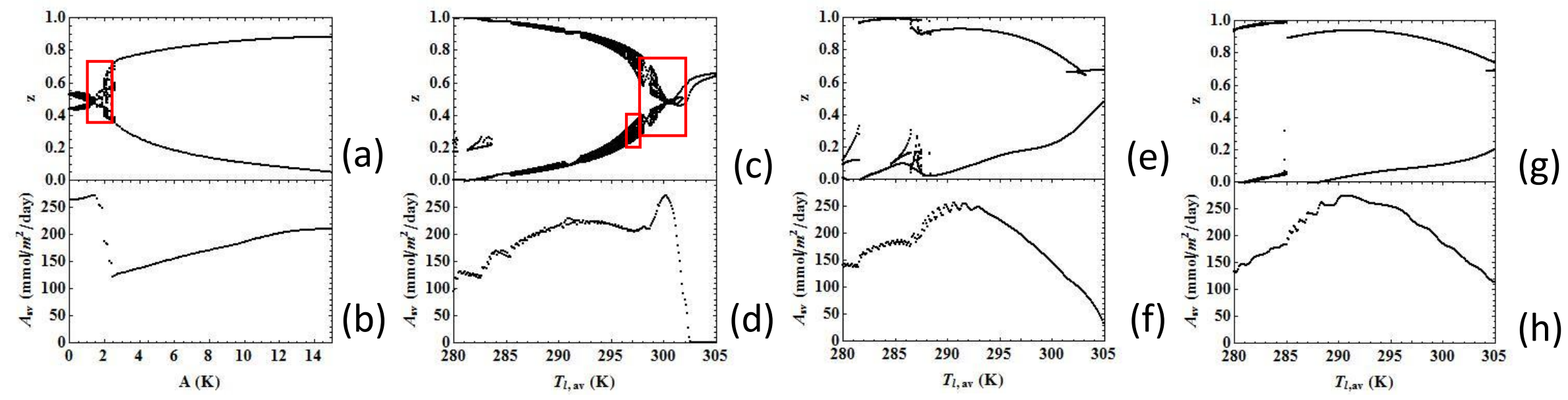
Figure 7
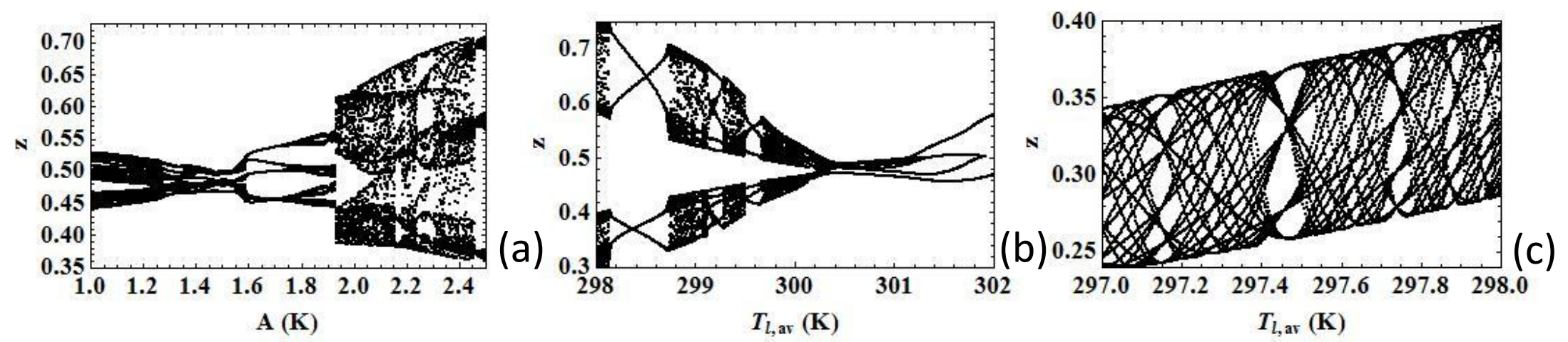


\section{Figure 8}
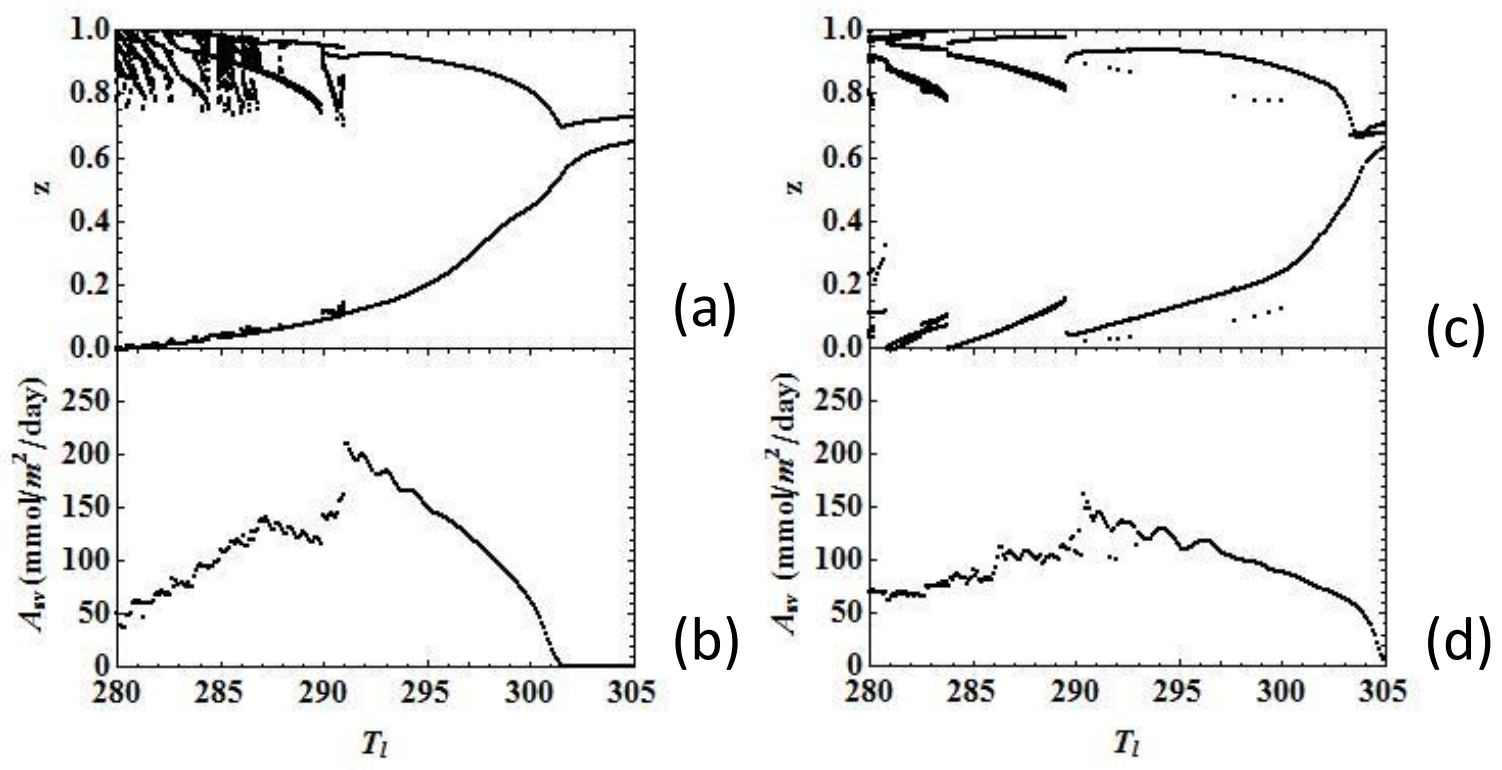
Figure 9
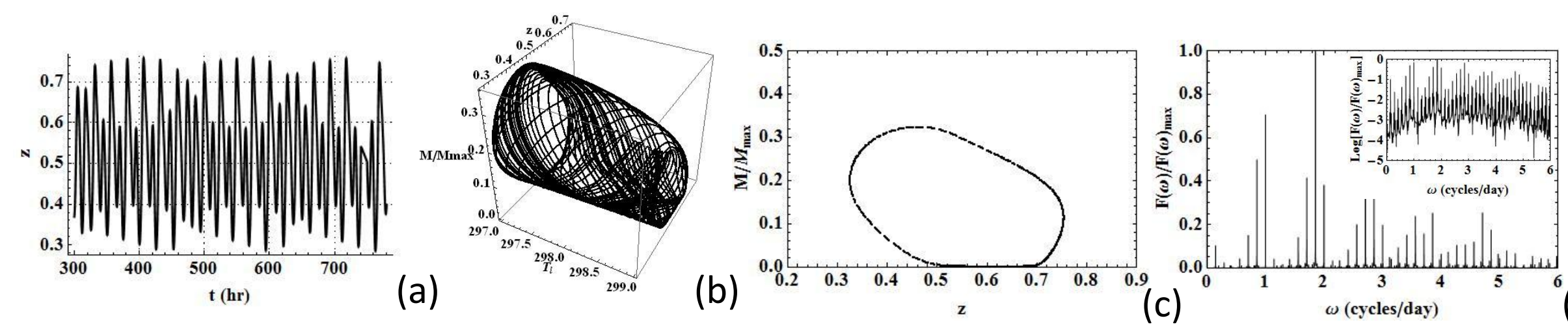

(d)
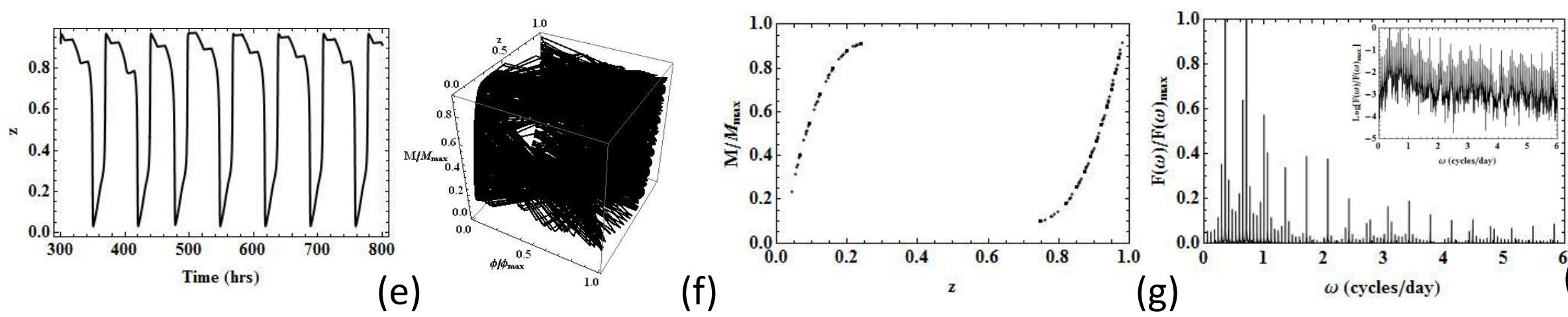

(h) 